Universidade de São Paulo

Instituto de Matemática e Estatística

\title{
Ciclos Canard e Bifurcação Homoclínica em uma Família a 3 Parâmetros de Campos de Vetores no Plano
}

Paulo Ricardo da Silva

Tese Submetida ao Curso de Pós-Graduação em Matemática para Obtenção do Título de Doutor em Matemática

Orientador

Prof. Dr. Jorge Manuel Sotomayor Tello

São Paulo, Agosto de 1997. 


\section{Ciclos Canard e Bifurcação Homoclínica em uma Família a 3 Parâmetros de Campos de Vetores no Plano}

Este exemplar corresponde à redação final da tese apresentada por Paulo Ricardo da Silva ,devidamente corrigida e aprovada pela pela comissão julgadora.

São Paulo, setembro de 1997.

Banca Examinadora:

Prof. Dr. Jorge Sotomayor Tello (Preseidente)

IME-USP

Prof. Dr. Clodoaldo Grotta Ragazzo

IME-USP

Prof. Dr. Robert Roussarie

UNIV. de DIJON

Prof. Dr. Ronaldo Alves Garcia

UFG

Prof. Dr. Marco Antonio Teixeira

IMECC-UNICAMP 


\section{AGRADECIMENTOS}

Aos amigos Patrícia, Víctor e Welby ; pelo apoio dado em todos os momentos.

Aos colegas Ana ,L.Fernando , Marcelo ,Mauro e Wania ;por acompanharem a elaboração deste trabalho.

Aos professores R.Garcia, C.Ragazzo, R.Roussarie e M.Teixeira; pelas suas críticas e sugestões.

A J.Sotomayor; pela confiança ,pelos estímulos, pelas críticas e pelas sugestões. 


\section{ÍNDICE}

Resumo 3

Abstract 4

Introdução 5

1- Pre lim inares e Principais Resultados 7

2-A Transformação de Lienard 24

3-As Singularidades do Sistema $X_{\varepsilon, b, c}:\left\{\begin{array}{c}x^{\prime}=y-\left(b x+c x^{2}-4 x^{3}+x^{4}\right) \\ y^{\prime}=\varepsilon\left(x^{2}-2 x\right)\end{array} \quad 29\right.$

4-Os Retratos de Fase do Sistema $X_{\varepsilon, b . c}$

5-A Desingularização de $X_{\varepsilon, b, c} \quad 41$

6-O Fenômeno Canard 53

$\begin{array}{ll}\text { Bibliografia } & 64\end{array}$ 


\section{RESUMO}

Neste trabalho consideramos a família a 3-parâmetros de campos de vetores no plano dada por

$$
\left\{\begin{array}{l}
x \prime=y \\
y^{\prime}=x^{2}+\mu+y\left(\nu_{o}+\nu_{1} x+x^{3}\right)
\end{array}\right.
$$

$\operatorname{com}\left(x, y, \mu, \nu_{o}, \nu_{1}\right) \in R^{2} \times R^{3}[\mathrm{DRS} 1]$.

Provamos que se $\mu \rightarrow-\infty$ então (C) é $C^{\circ}$-equivalente a (D) para $\varepsilon>0, \varepsilon \downarrow 0, b, c \in R$.

$$
\left\{\begin{array}{l}
x^{\prime}=y-\left(b x+c x^{2}-4 x^{3}+x^{4}\right) \\
y^{\prime}=\varepsilon\left(x^{2}-2 x\right)
\end{array}\right.
$$

Provamos que ocorre uma Bifurcação de Hopf de codimensão 1 quando $b=0$ e que quando $b=0, c=12$ e $\varepsilon>0$ então ocorre uma Bifurcacão de Hopf de codimensão 2 . Estudamos o Fenômeno Canard e a Bifurcação Homoclínica na família (D). Provamos que quando $\varepsilon \downarrow 0, b=0$ e $c=12$ os ciclos criados apresentam um pequeno diâmetro e mudam para um grande diâmetro rapidamente num sentido a ser esclarecido aqui. 


\section{ABSTRACT}

Let the 3-Parameter Family given by

$$
\left\{\begin{array}{l}
x^{\prime}=y \\
y^{\prime}=x^{2}+\mu+y\left(\nu_{0}+\nu_{1} x+x^{3}\right)
\end{array}\right.
$$

with $\left(x, y, \mu, \nu_{o}, \nu_{1}\right) \in R^{2} \times R^{3}[\mathrm{DRS} 1]$.

We prove that if $\mu \rightarrow-\infty$ then (A) is $C^{0}$-equivalent to (B) for $\varepsilon>0, \varepsilon \downarrow 0, b, c \in \mathbb{R}$.

$$
\left\{\begin{array}{l}
x^{\prime}=y-\left(b x+c x^{2}-4 x^{3}+x^{4}\right) \\
y^{\prime}=\varepsilon\left(x^{2}-2 x\right)
\end{array}\right.
$$

We prove that there exists a Hopf Bifurcation of Codimension 1 when $b=0$ and also that, if $b=0, c=12$ and $\varepsilon>0$ then there exists a Hopf Bifurcation of codimension 2. We study the "Canard Phenomenon" and the Homoclinic Bifurcation in the family (B). We show that when $\varepsilon \downarrow 0, b=0$ and $c=12$ the cycles created stay with "small size "and change to a "big size "very quickly, in a sense made precise here. 


\section{INTRODUÇĀO}

Consideremos $X_{n}$ um campo de vetores no plano tal que $(0.0)$ é uma singularidade com parte linear nilpotente. isto é $j^{1} X_{0}=y \frac{\partial}{\partial x}$.

Bogdanov mostrou .em [B-1] e [B-2], que, genericamente. todos os desdobramentos locais a 2 parâmetros de $X_{0}$ são topologicamente equivalentes. Os modelos para estes desdobramentos. conhecidos como cúspides de codimensão 2 (Bifurcação de Bogdanov-Takens) são:

$$
y \frac{\partial}{\partial x}+\left(x^{2}+\mu+y(\nu \pm x)\right) \frac{\partial}{\partial y}
$$

Com uma condição extra de degenerecência, a saber $j^{2} X=y \frac{\partial}{\partial x}+x^{2} \frac{\partial}{\partial y}$, Dumortier, Roussarie e Sotomayor provaram que, genericamente, todos os desdobramentos locais a 3-parâmetros, são topologicamente equivalentes. Os modelos para estes desdobramentos, conhecidos como cúspides de codimensão 3, [DRS-1], são:

$$
y \frac{\partial}{\partial x}+\left(x^{2}+\mu+y\left(\nu_{0}+\nu_{1} x \pm x^{3}\right)\right) \frac{\partial}{\partial y}
$$

Neste trabalho estudamos os ciclos limites de $\left(\mathrm{II}^{+}\right)$quando $\mu \rightarrow-\infty$.

Para a cúspide de codimensão 2 (I), tal estudo encontra-se em [B]. Boutat, além de estender o diagrama de bifurcação da cúspide de codimensão 2, para $\mu \rightarrow-\infty$, estudou o "Fenômeno Canard", ocorrendo com os ciclos limites. Basicamente ele provou que o ciclo criado numa bifurcação de Hopf permanece "pequeno" e muda para um tamanho "grande" muito rapidamente. Neste contexto entendemos por pequeno o ciclo próximo do foco e grande o ciclo com diâmetro próximo do diâmetro do laço onde ele desaparecerá em uma bifurcação homoclínica.

O "Fenômeno Canard" foi primeiramente estudado em [BCD] em termos de Análise Não Standard. Recentemente, uma interpretação geométrica deste fenômeno foi dada por Dumortier e Roussarie [DR1] utilizando variedades centrais e desingularização global.

Neste trabalho inicialmente mostramos que o estudo de (II ${ }^{+}$) quando $\mu \rightarrow-\infty$ é equivalente ao estudo de (III) quando $\varepsilon \downarrow 0, b, c \in \mathbb{R}$.

$$
\left\{\begin{array}{l}
x^{\prime}=y-\left(b x+c x^{2}-4 x^{3}+x^{4}\right) \\
y^{\prime}=\varepsilon\left(x^{2}-2 x\right)
\end{array}\right.
$$


Provamos que quando $c=12, b=0 \mathrm{e} \varepsilon>0$ ocorre uma bifurcação de Hopf de codimensão 2 e que os ciclos criados permanecem pequenos e muito rapidamente mudam para um grande tamanho.Estudamos a bifurcação homoclínica quando $\varepsilon \downarrow 0$. O limite dos pontos $(\varepsilon, b, c)$, onde ocorre a Bifurcação Homoclínica de codimensão 2 , é $(\varepsilon, b, c)=(0.0,4)$.

Em 1 apresentamos alguns conceitos e resultados preliminares e colocamos os resultados numa forma precisa. Em 2 efetuamos algumas mudanças de coordenadas, destacando-se a transformação de Liénard e colocamos $\left(\mathrm{II}^{+}\right)$na forma (III). Em 3 determinamos a bifurcação de Hopf ocorrendo em (III).

Em 4 estudamos o retrato de fase de (III) e em 5 e 6 estudamos o Fenômeno Canard e a bifurcação homoclínica. 


\section{1 - PRELIMINARES E PRINCIPAIS RESULTADOS}

Dizemos que $X_{\lambda}, \lambda \in \mathbb{R}^{k}$, dada por (1), é uma família a k-parametros de campos de vetores no plano, onde $m=(x, y) \in \mathbb{R}^{2} e a . b$ são funções $\left(^{\infty}\right.$.

$$
X_{\lambda}=a(m, \lambda) \frac{\partial}{\partial x}+b(m, \lambda) \frac{\partial}{\partial y}
$$

A família é dita local quando $m$ e $\lambda$ estão em alguma vizinhança da origem, em $\mathbb{R}^{2}$ e $\mathbb{R}^{k}$. respectivamente. $X_{\lambda}$ é dito um desdobramento ou uma deformação de $X_{0}$.

Dizemos que $X$ e $Y$ são $C^{0}$-equivalentes (ou topologicamente equivalentes) se existe $h$, um homeomorfismo em $\mathbb{R}^{2}$, levando X-órbitas em Y-órbitas e preservando a orientação.

Duas famílias $X_{\lambda}$ e $Y_{\mu}$ são ditas $C^{0}$-equivalentes por fibras se existem homeomorfismos $\mu=\phi(\lambda)$ e $h_{\lambda}$ tais que $\forall \lambda \in \mathbb{R}^{k}, h_{\lambda}$ é uma equivalência topológica entre $X_{\lambda}$ e $Y_{\phi(\lambda)}$.

O conjunto de bifurcação de $X_{\lambda}$ é o menor subconjunto fechado $\sum \subset \mathbb{R}^{k}$ tal que o tipo topológico de $X_{\lambda}$ é localmente constante em $\mathbb{R}^{k} \backslash \sum$.

Dizemos que $X$ é $C^{\infty}$-equivalente a $Y$ se $Y=g_{*} X$ onde $g_{*} X(x)=\left(d g_{x}\right)^{-1} X(g(x))$ e $g$ é um difeomorfismo fixando a origem.

Consideremos $\mathfrak{X}_{0}$ o conjunto dado por (2):

$$
\mathfrak{X}_{0}=\left\{X=P(x, y) \frac{\partial}{\partial x}+Q(x, y) \frac{\partial}{\partial y} \mid P, Q \in C^{\infty}, \quad P(0)=Q(0)=0\right\} .
$$

Dividimos $\mathfrak{X}_{0}$ em diferentes subconjuntos considerando as formas de Jordan de suas partes lineares na origem.

$$
\begin{aligned}
& T_{1}:\left\{\begin{array}{l}
\left(\begin{array}{ll}
a & b \\
0 & c
\end{array}\right) a \neq 0, c \neq 0 \text { e } b=0 \text { ou } a=c \neq 0 \text { e } b=1 \\
\left(\begin{array}{cc}
a & b \\
-b & a
\end{array}\right) \quad a \neq 0, b \neq 0
\end{array}\right. \\
& T_{2}:\left(\begin{array}{ll}
a & 0 \\
0 & 0
\end{array}\right) \quad a \neq 0
\end{aligned}
$$




$$
\begin{aligned}
& T_{3}:\left(\begin{array}{ll}
0 & -a \\
a & 0
\end{array}\right) a \neq 0 \\
& T_{4}:\left(\begin{array}{ll}
0 & 1 \\
0 & 0
\end{array}\right) \\
& T_{5}:\left(\begin{array}{ll}
0 & 0 \\
0 & 0
\end{array}\right)
\end{aligned}
$$

Definimos $W_{i}$ como sendo o subconjunto de $\mathfrak{X}_{0}$ tal que em $W_{i}$ a forma de Jordan da parte linear é linearmente equivalente a dada em $T_{i}$. Assim $\mathfrak{X}_{0}=W_{1} \cup W_{2} \cup W_{3} \cup W_{4} \cup W_{5} . W_{1}$ é o conjunto dos campos cuja singularidade $(0,0)$ é hiperbólica. $W_{2}$ é o conjunto dos campos com singularidade $(0,0)$ do tipo sela-nó. $W_{3}$ é o conjunto dos campos com singularidade do tipo Hopf. Estamos interessados em $W_{4}^{\prime}$ onde a singularidade $(0,0)$ tem parte linear nilpotente.

Se $X_{0} \in W_{4}$ então podemos escrever, conforme [DRS-1], $j^{2} X_{0}$ na forma:

$$
j^{2} X_{0}=y \frac{\partial}{\partial x}+\left(a x^{2}+b x y\right) \frac{\partial}{\partial y}
$$

Se $a \neq 0$ dizemos que $X_{0} \in W_{4}$ tem singularidade tipo cúspide. Para o caso $a=0$ indicamos [DRS-2].

Genericamente temos que $a b \neq 0 \mathrm{e}$, conforme $[\mathrm{D}]$, temos que $X_{0}$ é $\mathrm{C}^{\infty}$-equivalente a

$$
y \frac{\partial}{\partial x}+\left(x^{2} \pm x y\right) \frac{\partial}{\partial y}
$$

A bifurcação de Bogdanov-Takens [RW] é a bifurcação da família $X_{\lambda}, \lambda=(\mu, \nu)$, dada por

$$
y \frac{\partial}{\partial x}+\left(x^{2}+\mu+y(\nu \pm x)\right) \frac{\partial}{\partial y}
$$

Se $\mu>0$ o campo de vetores não admite singularidades e seu retrato de fase é trivial. Se $\mu=0$ e $\nu \neq 0$ temos uma bifurcação do tipo sela-nó isto é, quando os parâmetros cruzam a linha $\mu=0$ surgem duas singularidades, um foco (ou nó) e uma sela. A curva $H$, onde a divergència anula-se no foco, é a curva de bifurcação genérica de Hopf (isto é. na forma normal 
em coordenadas polares, o campo começa com termos de ordem 3 ). Ao cruzar a linha $H$ surge um ciclo limite que irá desaparecer na curva $L$ onde temos uma órbita homoclínica (isto é . uma sela que tem uma separatriz instável coincidindo com uma separatriz estável ).

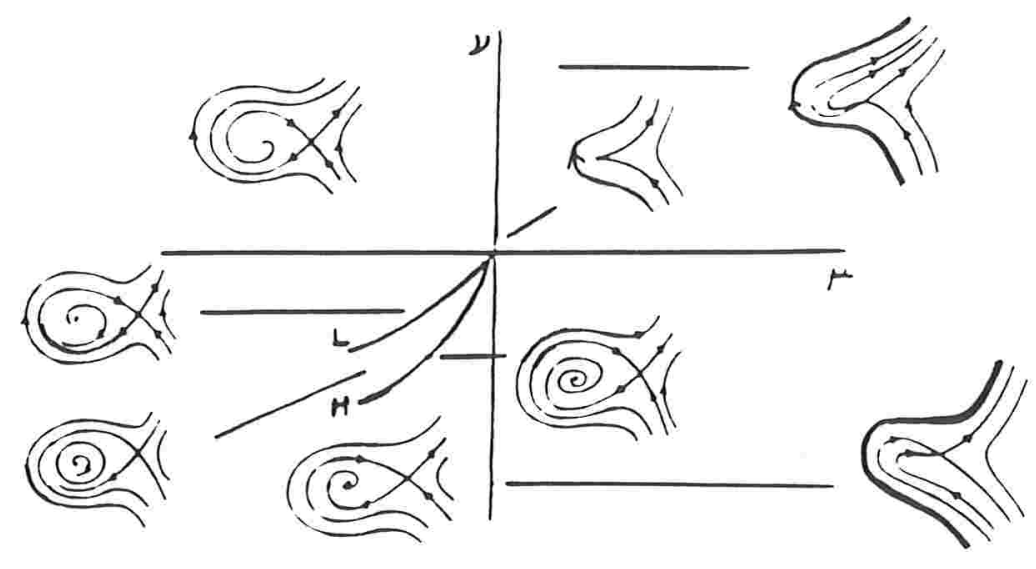

Figura 1:Diagrama de Bifurcação de $\left(6^{-}\right)$

Em [DR2] encontramos a prova de que o diagrama de bifurcação de (6) pode ser estendido para os parâmetros $\mu$ e $\nu$ arbitrários.

Boutat, em [B], estudou o comportamento dos ciclos de (6) quando $\mu \rightarrow-\infty$. Efetuando algumas mudanças de coordenadas temos que (6+), onde $\varepsilon \downarrow 0$ e $a \in R$, é equivalente a

$$
X_{\varepsilon, a}:\left\{\begin{array}{l}
x^{\prime}=y-\frac{x^{2}}{2} \\
y^{\prime}=\varepsilon\left(2(a-x)-(x-a)^{2}\right)
\end{array}\right.
$$

Se $\varepsilon=0$ o retrato de fase de $X_{0, a}$ é dado por

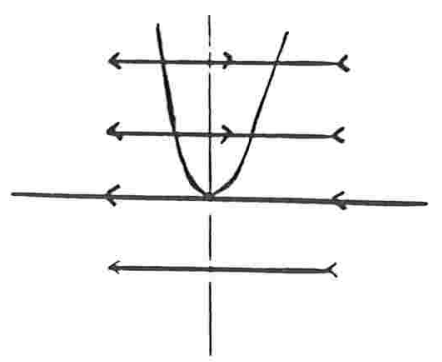

Figura 2:Retrato de Fase de $X_{0 . a}$ 
Os pontos ao longo da curva $y=\frac{x^{2}}{2}$, exceto $(0,0)$, são normalmente hiperbólicos. Para $\varepsilon \neq 0$. obtemos duas singularidades $\left(a \cdot a^{2} / 2\right)$ e $\left(a-2, \frac{(a-2)^{2}}{2}\right)$. A primeira é um nó ou foco atrator para $a>0$ e um nó ou foco repulsor para $a<0$. A segunda é um ponto de sela.

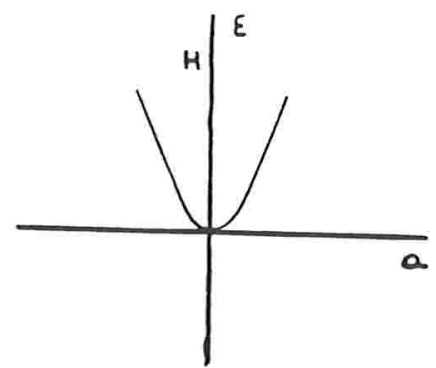

Figura 3:Bifurcação de Hopf de $X_{\varepsilon . a}$

Para $\varepsilon>0$ suficientemente pequeno os retratos de fase de $X_{\varepsilon, a}$ são dados por 

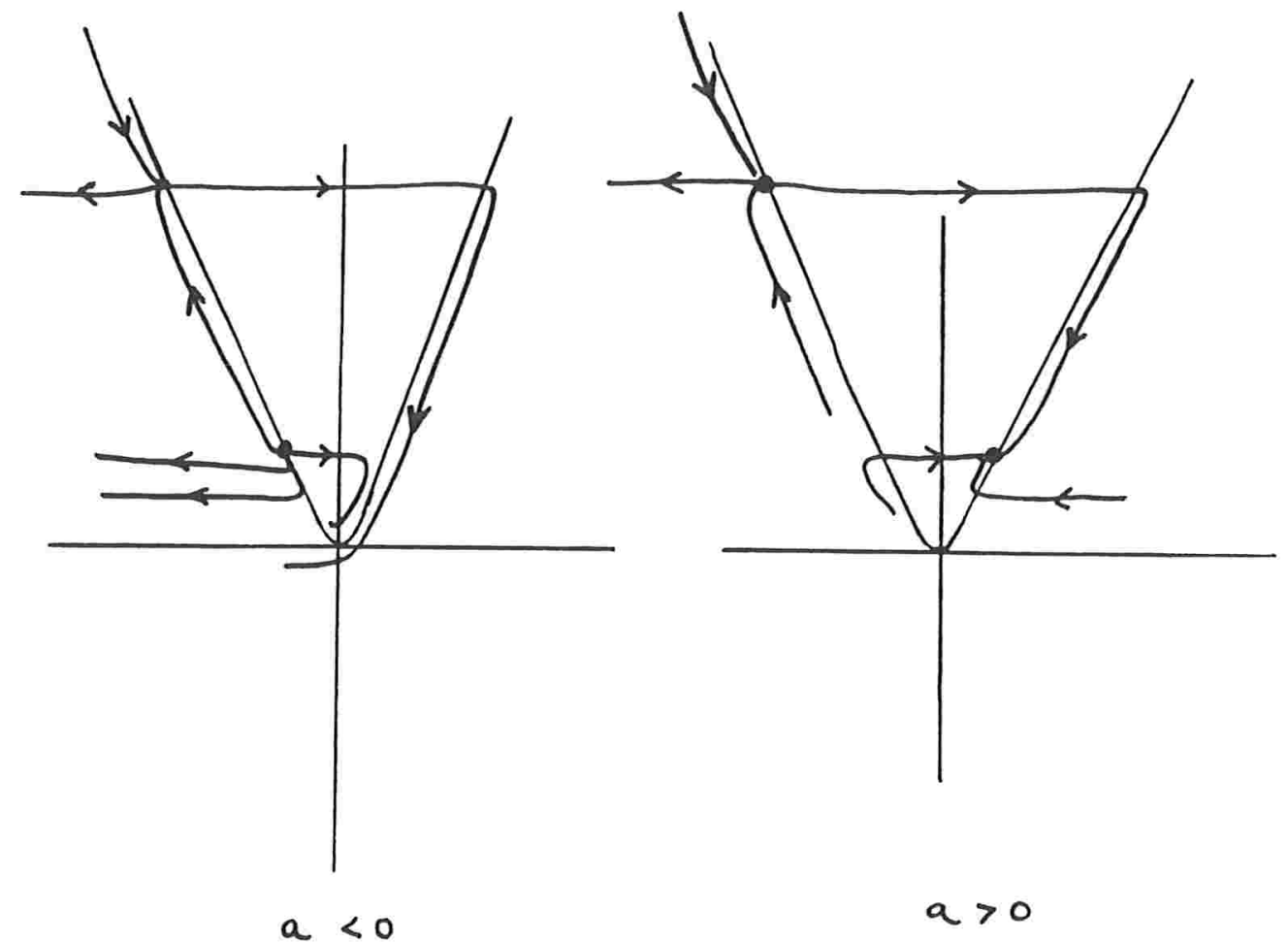

Figura 4: Retratos de Fase de $X_{\varepsilon, a}$ para $\varepsilon \downarrow 0$ 
Quando $a=0$ ocorre uma bifurcação genérica de Hopf e um ciclo limite surge. Boutat analisou o que ocorre com o ciclo quando $\varepsilon \downarrow 0$.

Chamamos de Limite de Conjunto Periódico ( $L C P$ ) um possível limite de uma seqüência de ciclos limites.Precisamente, dizemos que $\Gamma \in R^{2}$,compacto, é um $L C P$ de $X_{\varepsilon, a}$ se $\exists \varepsilon_{n} \downarrow 0$ , $a_{n} \downarrow 0$ e $\Gamma_{n} \subset R^{2}$ ciclo limite de $X_{\varepsilon_{n}, a_{n}}$ tais que $\Gamma_{n} \rightarrow \Gamma$, segundo a distância de Hausdorff. Lembramos que a distância de Hausdorff entre dois compactos $\mathrm{A}$ e $\mathrm{B}$ do plano é dada por $d(A, B)=\max _{x \in A, y \in B}\{d(x, B), d(y, A)\}$. É fácil ver que os possíveis LCP's de $X_{\varepsilon, a}$ são dados pela figura :

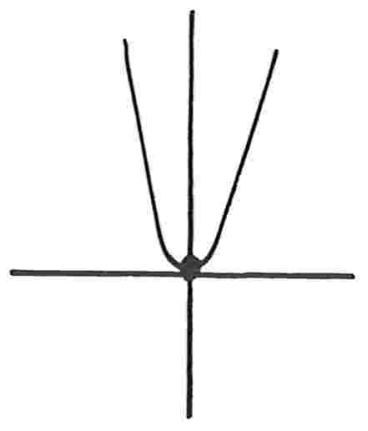

$\Gamma_{p}$

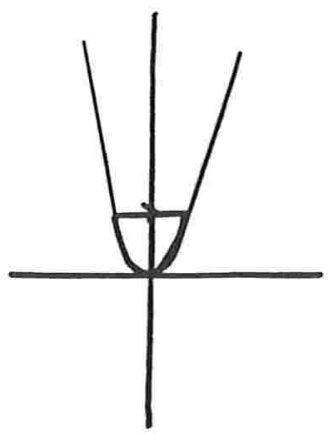

Figura 5:LCP's de $X_{\varepsilon, a}$

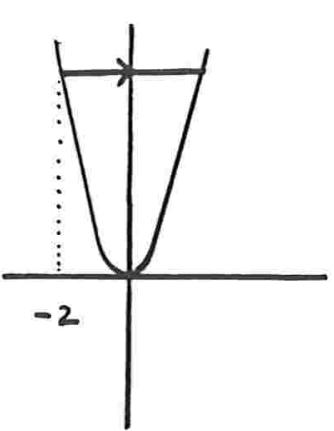

$\Gamma_{G}$ 
Os LCP's são conhecidos por Ciclos Canard, ao que tudo indica, devido ao fato dos ciclos limites da equação de Van der Pol possuirem um aspecto semelhante a um pato [E].

Definiçāo : Seja $X_{\lambda}=X(\lambda, p)$ uma familia a $k$-parâmetros de campos de vetores no plano. Dizemos que ocorre o Fenômeno Canard na familia $X_{\lambda}$ se:

a)Existem sequências $\alpha_{n}$ e $\beta_{n}$ em $R^{k}$ tais que $\left\|\alpha_{n-} \beta_{n}\right\| \longrightarrow 0$.

b)Existe $h_{n}$, homeomorfismo, levando órbitas de $X_{\alpha_{n}}$ em órbitas de $X_{\beta_{n}}$.

c) $X_{\alpha_{n}}$ admite um ciclo limite $C_{n}$ e $X_{\beta_{n}}$ um ciclo limite $D_{n}$ tais que $h_{n}\left(C_{n}\right)=D_{n}$.

d)Existe um número positivo $r$ tal que diam $D_{n} \longrightarrow r$ e diam $C_{n} \longrightarrow 0$.

O Fenômeno Canard tem sido caracterizado através do contato flat entre gráficos no espaço de parâmetros $([B],[D R 1],[E])$.

Boutat empregou a técnica recente de Desingularização Global e Variedades Centrais, introduzida por Dumortier e Roussarie [DR1] e provou o seguinte teorema:

Teorema 1 Sejam $\Gamma_{P}$ e $\Gamma_{G}$ os LCP's indicados na figura 5 .Existem curvas $a_{1}=a_{1}(\varepsilon)$, $a_{2}=a_{2}(\varepsilon), a_{1}$ e $a_{2} \in C^{\infty}$, definidas para $\varepsilon>0$ com $a_{1}(0)=a_{2}(0)=0$, tendo um contato flat com o eixo $\varepsilon$, tais que $X_{\varepsilon, a_{1}(\varepsilon)}$ admite um ciclo limite $\Gamma_{\varepsilon}, X_{\varepsilon, a_{2}(\varepsilon)}$ admite um ciclo $\widetilde{\Gamma_{\varepsilon}}$,com $\Gamma_{\varepsilon} \rightarrow \Gamma_{G}$ e $\widetilde{\Gamma_{\varepsilon}} \rightarrow \Gamma_{P}$, segundo a distância de Hausdorff, quando $\varepsilon \downarrow 0$.

As curvas $a_{1}(\varepsilon)$ e $a_{2}(\varepsilon)$ são chamadas de Curvas Canard.

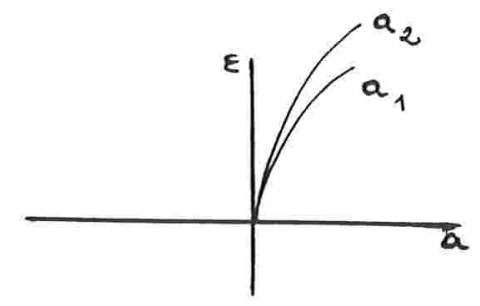

Figura 6:Curvas Canard 
Interpretamos o teorema 1, geometricamente, como um crescimento muito rápido do ciclo criado em $\{a=0\}$ quando $\varepsilon \downarrow 0$. Segundo a nossa definição ocorre o Fenômeno Canard na família $X_{\varepsilon, a}$, estudada por Boutat.De fato basta considerarmos $\beta_{n}=\left(\frac{1}{n}, a_{1}\left(\frac{1}{n}\right)\right)$ e $\alpha_{n}=\left(\frac{1}{n}, a_{2}\left(\frac{1}{n}\right)\right)$.

O ponto de partida deste trabalho é a cúspide de codimensão 3 , isto é, o desdobramento de $X_{0} \in W_{4}$ tal que $j^{2} X_{0}$ é do tipo

$$
j^{2} X_{0}=y \frac{\partial}{\partial x}+x^{2} \frac{\partial}{\partial y}
$$

Consideremos a família a 3 parâmetros em dada por:

$$
\left\{\begin{array}{l}
x^{\prime}=y \\
y^{\prime}=x^{2}+\mu+y\left(\nu_{0}+\nu_{1} x+x^{3}\right)
\end{array}\right.
$$

Esta família é o desdobramento genérico da cúspide de codimensão 3 [DRS-1]. O diagrama de bifurcação para $\left(x, y, \mu, \nu_{0}, \nu_{1}\right)$ em uma vizinhança da origem $(0,0,0,0,0)$ está representado nas figuras 7 e 8 . Para $\mu$ positivo o retrato de fase de (9) é trivial já que o sistema não admite singularidades.
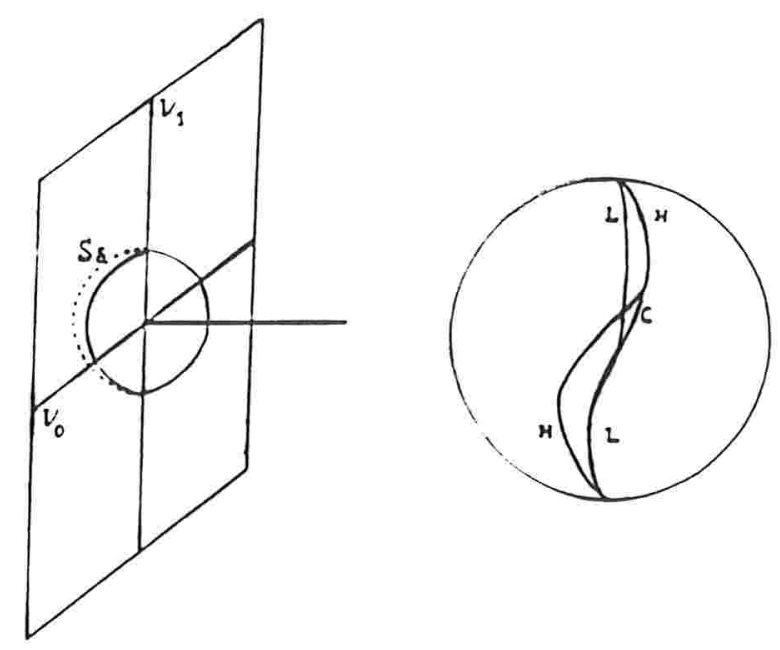

Figura 7: A Interseç̧ão do Diagrama de (9) $\operatorname{com} S_{\delta}$ 


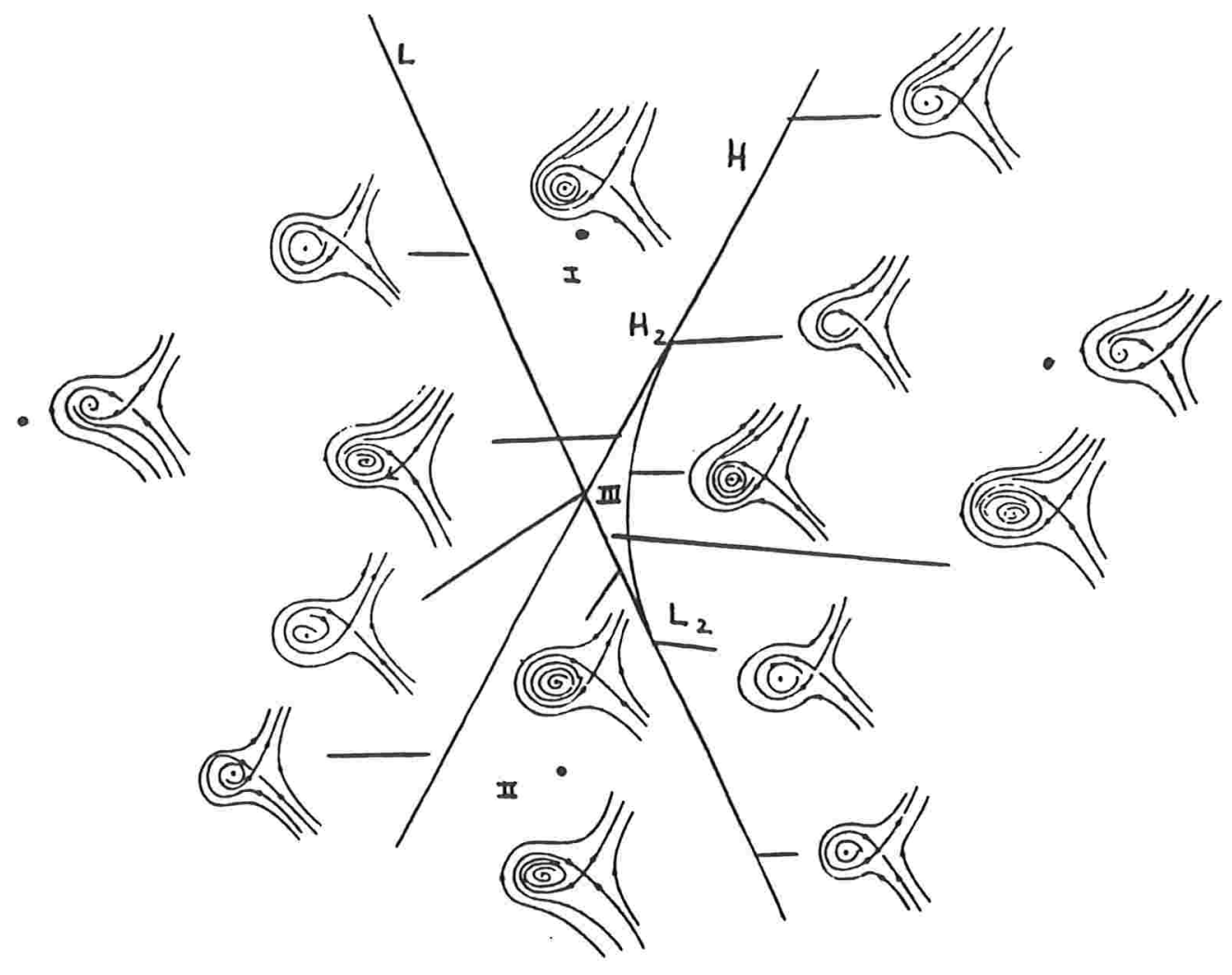

Figura 8 :Retratos de Fase de (9)

15 
A figura (7) mostra a intersecção do diagrama de bifurcação com a esfera $S_{\delta}=$ $\left\{\left(\mu, \nu_{0}, \nu_{1}\right) \mid \mu^{2}+\nu_{0}^{2}+\nu_{1}^{2}=\delta^{2}\right\}$, onde $\delta>0$ é suficientemente pequeno. Existem um ciclo limite repulsor e um foco atrator em (I) e existem um ciclo limite atrator e um foco repulsor em (II). Para valores do parâmetro em (III) existem dois ciclos limites sendo o interno atrator e o outro repulsor. No plano dado por $\mu=0$ ocorre a bifurcação do tipo Sela-Nó isto é, sela e nó colapsam e desaparecem para $\mu$ positivo. A superfície $H$ representa a Bifurcação de Hopf e a curva $H_{2}$ a Bifurcação de Hopf de codimensão 2. Na superfície $L$ ocorre a Bifurcação Homoclínica e na curva $L_{2}$ a Bifurcação Homoclínica de codimensão 2.

O objetivo deste trabalho é estudar o comportamento dos ciclos limites quando $\mu \rightarrow-\infty$

Em 2 realizamos algumas mudanças de coordenadas, destacando-se a transformação de Liénard, e provamos que o conjunto de bifurcação de (9), para $\mu \rightarrow-\infty$, é equivalente ,quando $\varepsilon \downarrow 0, b, c \in \mathbb{R}$, ao conjunto de bifurcação de

$$
\left\{\begin{array}{l}
x^{\prime}=y-F_{b, c}(x) \\
y^{\prime}=\varepsilon\left(x^{2}-2 x\right)
\end{array}\right.
$$

Denotamos por $F_{b, c}$ a função real dada por

$$
F_{b, c}(x)=b x+c x^{2}-4 x^{3}+x^{4}
$$

Em 2 estudamos o conjunto de bifurcação da família de funções dadas por (11). Tal conjunto está representado na figura 


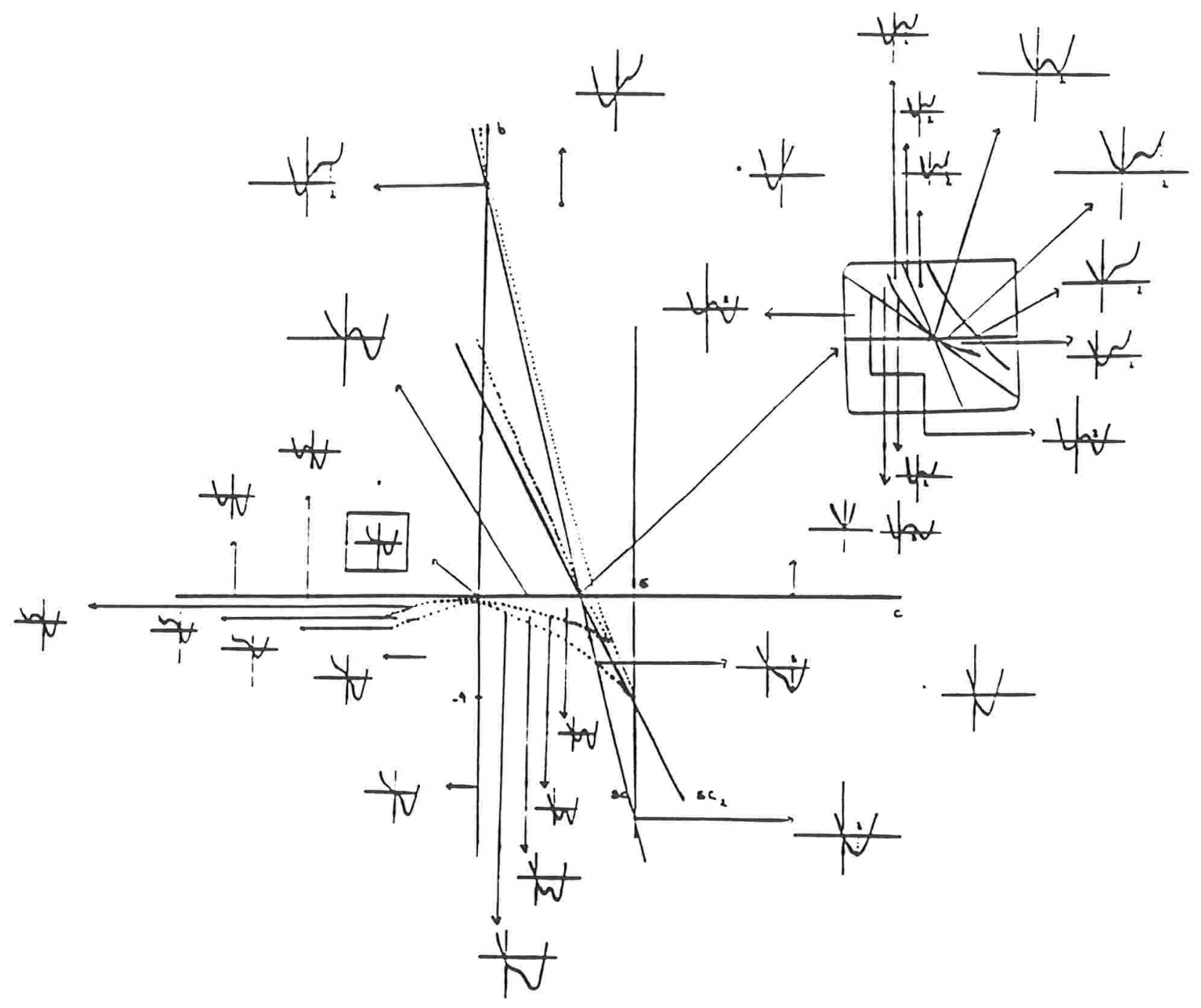

Figura 9: Diagrama de Bifurcação de $F_{b, c}$ 
Em 3 estudamos as singularidades do sistema (10). Quando $\varepsilon=0$ e $(b, c)$ está fixo as singularidades encontram-se na curva $L_{b . c}$ onde $L_{b . c}$ é dada por

$$
L_{b . c}=\left\{(x, y) \mid y=F_{b . c}(x)\right\}
$$

Os pontos em $L_{b . c}$, que não são críticos, são pontos singulares normalmente hiperbólicos. $\mathrm{O}$ retrato de fase está representado na figura

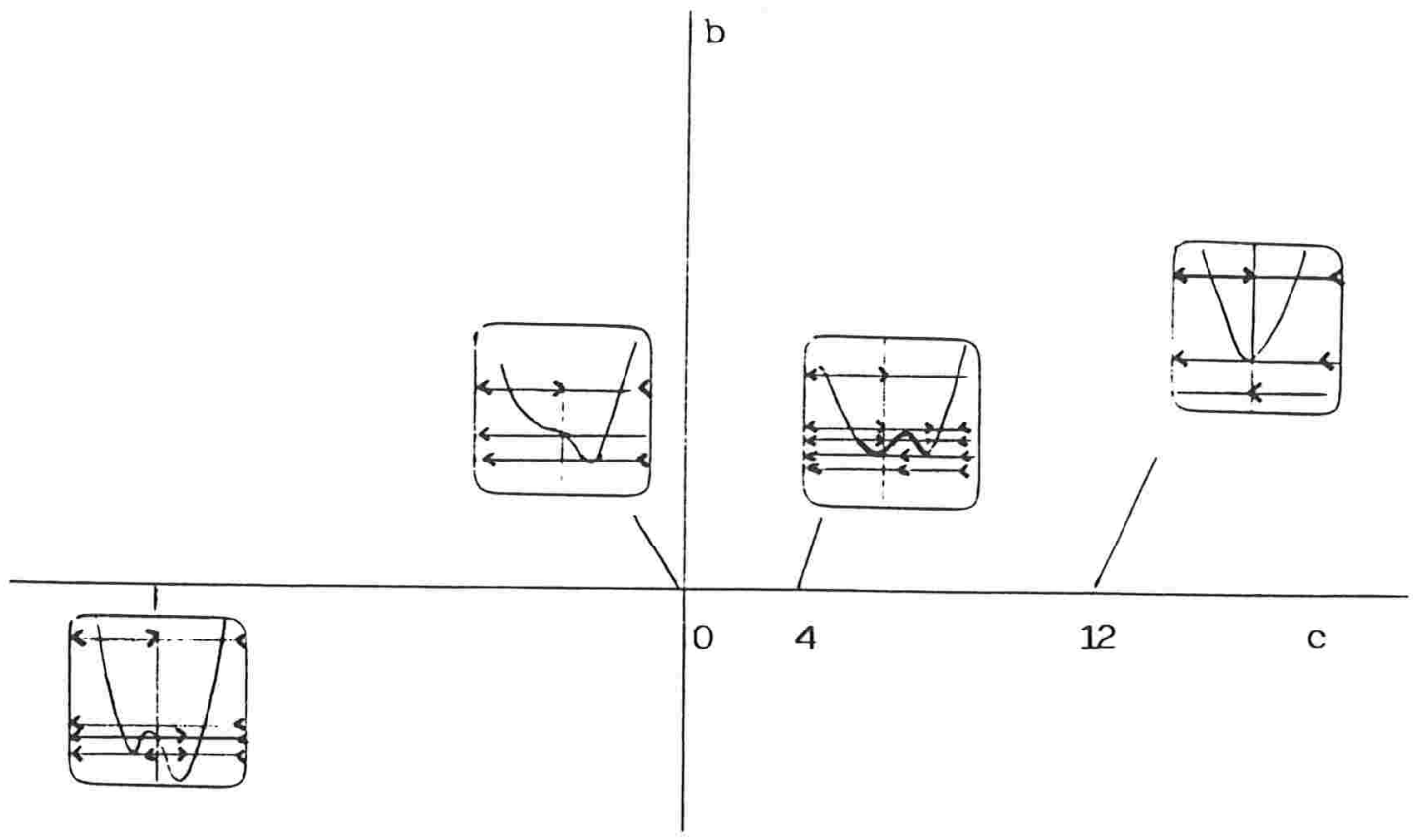

Figura 10:Retrato de Fase Quando $\varepsilon=0$ 
Para $\varepsilon$ positivo $(10)$ admite 2 singularidades: $(0,0)$ e $\left(2, F_{b, c}(2)\right)$.

$$
\begin{array}{cll}
0<\varepsilon \leq b^{2} / 8 & & \\
(0,0) & b>0 & \text { Nó Atrator } \\
& b<0 & \text { Nó Repulsor } \\
\left(2, F_{b . c}(2)\right) & b, c \in R & \text { Sela } \\
\varepsilon>b^{2} / 8 & & \\
(0,0) & b>0 & \text { Foco Atrator } \\
& b<0 & \text { Foco Repulsor } \\
\left(2, F_{b . c}(2)\right) & b, c \in R & \text { Sela }
\end{array}
$$

Tabela I : Classificação das Singularidades de (10)

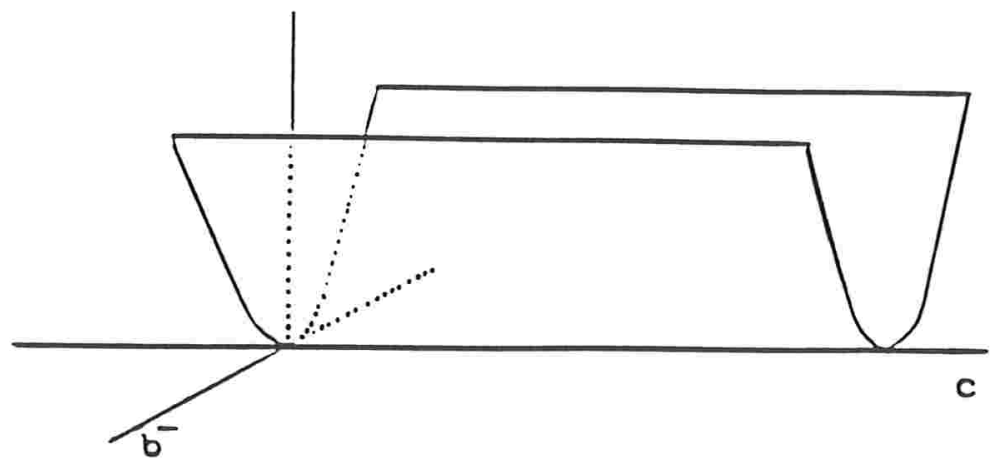

Figura 11: A Superfície $\varepsilon=\frac{b^{2}}{8}$ 
Devemos ter um cuidado maior para classificar a singularidade $(0,0)$ quando $b=0$ pois neste caso o autor-valor associado apresenta parte real nula. Provamos em 3 que ocorre uma Bifurcação de Hopf em $H=\{b=0, c \neq 12\}$ e uma Bifurcação de Hopf de codimensão $2 \mathrm{em}$ $H_{2}=\{b=0, c=12\}$. Provamos que surgem 2 ciclos limites em $\{b>0, c<12\}$ sendo o interno repulsor e o outro atrator.

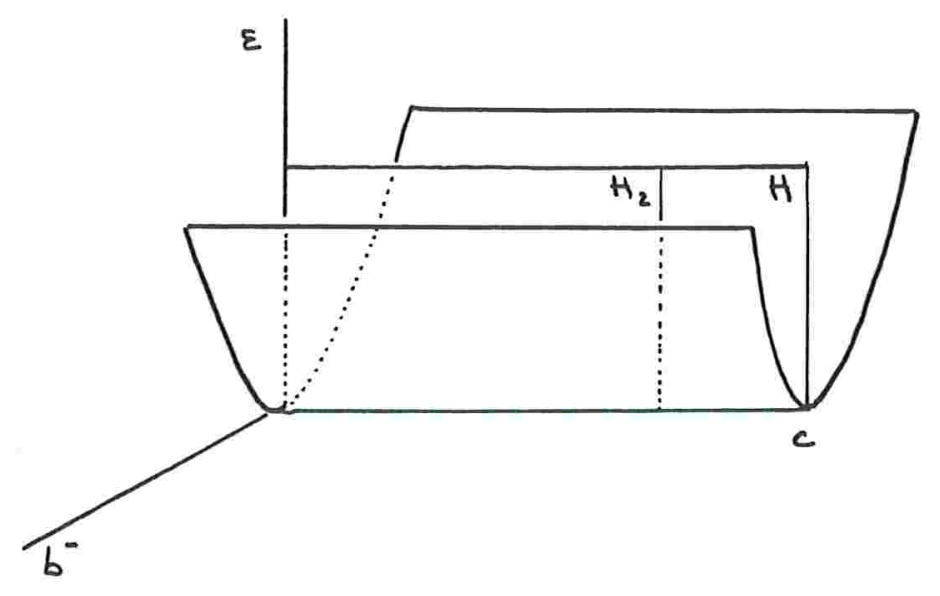

Figura 12: Bifurcação de Hopf em (10)

Estudamos o que ocorre com os ciclos criados em $H_{2}$ quando $\varepsilon \downarrow 0$. Quando $\varepsilon \downarrow 0$ os LCP's são aqueles onde $y$ é constante ou $y=F_{b, c}(x)$.

Os principais resultados deste trabalho são os seguintes teoremas:

Teorema 2 Consideremos a familia dada por (10). Seja $\Gamma$ o LCP ilustrado na figura 13. Existe uma superficie $S=\{b=\varphi(\sqrt{\varepsilon}, c)\}, \varphi \in C^{\infty}, \varphi(0, c)=0,(\varepsilon, b, c) \in v i z$ $(0,0,12)$, tal que $(\varepsilon, b, c) \in S$ então $(10)$ admite um ciclo $\left.\Gamma_{(}(\varepsilon, b, c), \Gamma_{(} \varepsilon, b, c\right) \rightarrow \Gamma$ segundo a distância de Hausdorff. quando $(\varepsilon, b, c) \rightarrow(0,0,12)$. 
A superfície $\mathrm{S}$ será chamada de Superfície Canard.

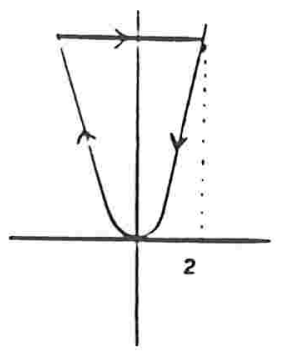

Figura 13:Ciclo Canard

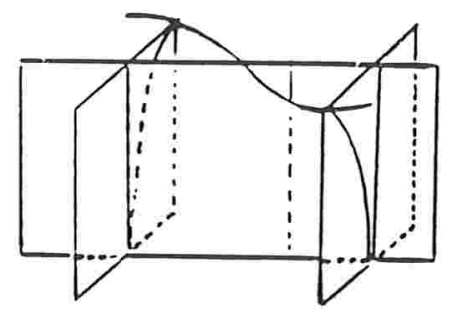

Figura 14:Superfície Canard 
Teorema 3 Consideremos a familia dada por (10) e ( $\varepsilon, b, c)$ em uma vizinhança de $(0,0,4)$. Existe uma superficie $L=\{b=v(\varepsilon, c)\}, v^{\prime \prime} \in C^{\infty} .(0,0,4) \in$ L. tal que para $(\varepsilon . b, c) \in L$. duas separatrizes do ponto de sela $\left(2, F_{b . c}(2)\right)$ formam uma órbita homoclínica. Em. $£_{2}=S C: \cap L$. $S C=\{b=-4 c+16\}$.temos uma órbita homoclínica de codimensão 2. Quando $\varepsilon \downarrow 0$ os pontos em $\AA_{2}$ convergem para $(\varepsilon, b, c)=(0,0,4)$.

Em 6 provamos que esta superfície é tal que para $b<0$, está à direita do plano, isto é , para $b<0, X_{\varepsilon, b, c}$ admite uma órbita homoclínica atratora. Em $\mathcal{L}_{1}=L \cap H$ temos uma órbita homoclínica atratora e um foco fraco repulsor. A partir de $\mathcal{L}_{2}, L$ está à esquerda do plano $S C$. isto é , $\mathrm{X}_{\varepsilon, b, c}$ admite uma órbita homoclínica repulsora.

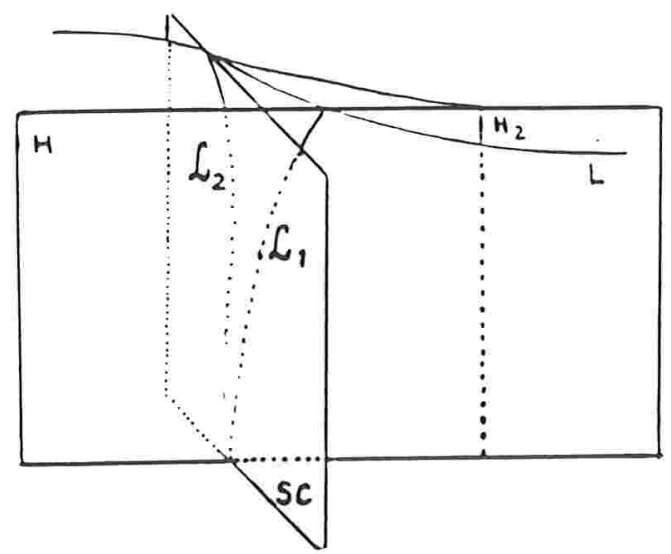

Figura 15:Diagrama de Bifurcação de $X_{\varepsilon, b . c}$ 
Lembramos que uma órbita homoclínica é dita de codimensão 2 quando a derivada da transformação de retorno, definida num dos lados da separatriz . for não nula .

As provas destes teoremas são dadas em 5 e 6 . Usamos o método introduzido em [DR1] que consiste basicamente na Desingularização Global e em Variedades Centrais.

Em 4 estudamos os retratos de fase de (10). Provamos que quando $\varepsilon \downarrow 0$ os valores dos parâmetros para os quais $X_{\varepsilon, b, c}$ admite ciclos limites convergem para $\{b=0\}$.

Os teoremas 2 e 3 mostram que quando $\varepsilon \downarrow 0$ os ciclos limites criados na Bifurcação de Hopf mudam para um grande tamanho e em seguida desaparecem em uma Bifurcação Homoclínica.

No final de 6 indicamos alguns problemas em aberto que podem ser estudados. 


\section{2 - A TRANSFORMAÇĀO DE LIÉNARD}

Consideremos $X_{\mu, \nu_{0}, \nu_{1}}$ dado por (9). Começamos com a seguinte transformação:

$$
\begin{array}{ll}
x=t^{2} x_{1} & \mu=-t^{4} \\
y=t^{3} y_{1} & \nu_{0}=t^{6} \nu_{0}^{\prime} \\
\nu_{1} & =t^{4} \nu^{\prime}{ }_{1} \\
& T=t^{5}
\end{array}
$$

Dividindo por $t$ e voltando a chamar $x, y, \nu_{0}, \nu_{1}$ obtemos

$$
\left\{\begin{aligned}
x^{\prime} & =y \\
y^{\prime} & =x^{2}-1+T y\left(\nu_{0}+\nu_{1} x+x^{3}\right)
\end{aligned}\right.
$$

Efetuando a transformação de Liénard (15) e denotando novamente por $x$ e $y$ obtemos (16)

$$
\begin{aligned}
& \left\{\begin{aligned}
T y_{1} & =\int T\left(\nu_{0}+\nu_{1} x+x^{3}\right) d x-y \\
x_{1} & =x
\end{aligned}\right. \\
& \left\{\begin{aligned}
x^{\prime} & =T\left(\nu_{0} x+\frac{\nu_{1}}{2} x+\frac{x^{4}}{4}\right)-T y \\
y^{\prime} & =-\frac{1}{T}\left(x^{2}-1\right)
\end{aligned}\right.
\end{aligned}
$$

A transformação de Liénard (15) é um difeomorfismo do plano $(x, y)$ no plano $\left(x_{1}, y_{1}\right)$.

Multiplicando por $\frac{-1}{T}$ e denotando $\varepsilon=\frac{1}{T^{2}}$ obtemos

$$
\left\{\begin{array}{l}
x^{\prime}=y-\frac{1}{4}\left(4 \nu_{0}+2 \nu_{1} x^{2}+x^{4}\right) \\
y^{\prime}=\varepsilon\left(x^{2}-1\right)
\end{array}\right.
$$

Multiplicando por 4 , fazendo $y_{1}=4 y, \nu_{0}^{\prime}=4 \nu_{0}, \nu_{1}^{\prime}=2 \nu_{1}, \varepsilon^{\prime}=\frac{1}{16} \varepsilon$ e voltando a chamar 
$x, y, \nu_{0}, \nu_{1}, \varepsilon$ obtemos

$$
\left\{\begin{array}{l}
x^{\prime}=y-\left(\nu_{0} x+\nu_{1} x^{2}+x^{4}\right) \\
y^{\prime}=\varepsilon\left(x^{2}-1\right)
\end{array}\right.
$$

Efetuando a mudança (19) e voltando a chamar $x$ e $y$ obtemos (10)

$$
\begin{aligned}
& x_{1}=x+1 \\
& y_{1}=y-1+\nu_{0}-\nu_{1} \\
& b=\nu_{0}-2 \nu_{1} \\
& c=\nu_{1}-6
\end{aligned}
$$

Vamos estudar a função dada por (11).

Proposiçāo $4 F_{b, c}: \mathbb{R} \longrightarrow \mathbb{R}$ dada por (11) admite um zero em $x_{0}=0$ e temos:

a) Se $b=0$ então $F_{b, c}$ admite um zero para $c>4,2$ zeros para $c=4$ ou $c=0$ e 3 zeros para $c<0$ ou $0<c<4$.

b) A equação (20) define uma cúspide no plano $(b, c)$. A cúspide é tangente ao eixo $c$ em. $(b, c)=(0,0)$, cruza o eixo c em $(b, c)=(0,4)$ e cruza o eixo b em $(b, c)=(9.48 \ldots, 0)$.

$$
\left(\frac{3 c-16}{9}\right)^{3}+\left(\frac{-36 c+128-27 b}{54}\right)^{2}=0
$$

c) A cúspide e o eixo c particionam o plano $(b, c)$ definindo o número de zeros de $F_{b, c}$ ( figura 16).

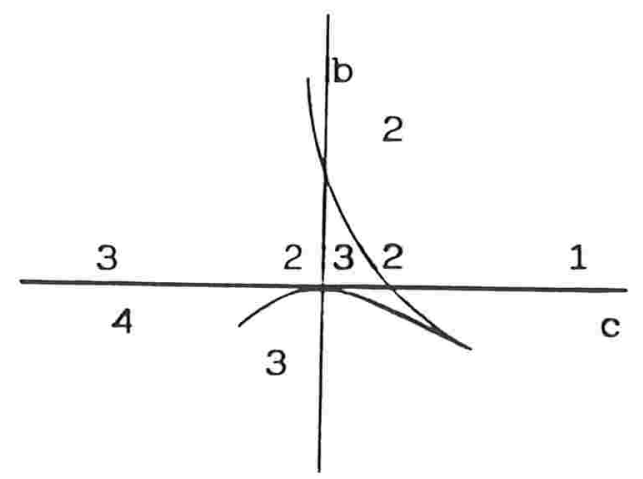

Figura 16:Zeros de $F_{b, c}$

\section{Demonstraçāo:}

A equação $(20)$ é a expressão $D=0$ onde $D$ é o discriminante de $\frac{F_{b . c}(x)}{x}=0 . \square$ 
Proposiçāo 5 Seja $F_{b . c}: \mathbb{R} \rightarrow \mathbb{R}$ dada por (11). Temos:

a) $S e b=0$ então $F_{b . c}$ admitc 3 pontos críticos quando $c<0$ ou $0<c<4.5 .1$ ponto crítico quando $c>4.5$ e 2 pontos críticos se $c=0$ ou $c=4.5$.

b) A equação 21 define uma cúspide no plano $(b, c)$ tangente ao eixo c em $(0,0)$. cruzando-o em $(0.4 .5)$, cruzando o eixo b em (16,0). tendo como vértice $(-4.6)$ e particionando, junto com o eixo $c, o(b, c)$-plano, definindo o número de pontos críticos de $F_{b, c}$.

$$
\left(\frac{c-6}{6}\right)^{3}+\left(\frac{-2 c-b+8}{8}\right)^{2}=0
$$

c) Se $c \geq 6$ então $F_{b, c}$ não admite inflexões e se $c<6$ então $F_{b, c}$ admite 2 inflexões. Além disso um ponto de inflexão será crítico se e somente se $(b, c)$ estiver em (21).

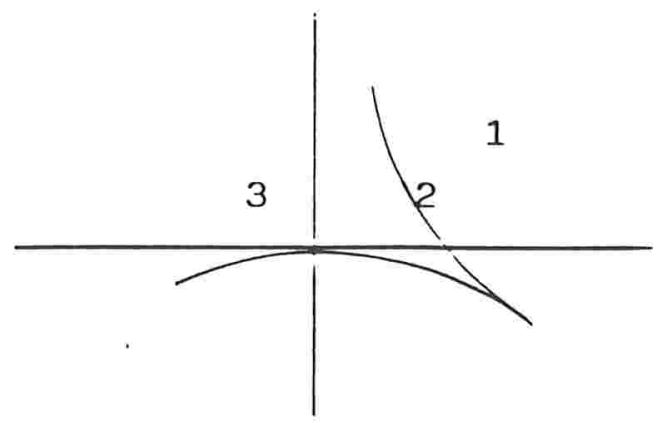

Figura 17: Pontos Críticos de $F_{b . c}$

\section{Demonstraçāo:}

A equação (21) é a expressão $D=0$ onde $D$ é o discriminante de $F_{b, c}^{\prime}(x)=0$.

As figuras 9 e 18 e a tabela II resumem as informações das proposições 4 e 5 . 


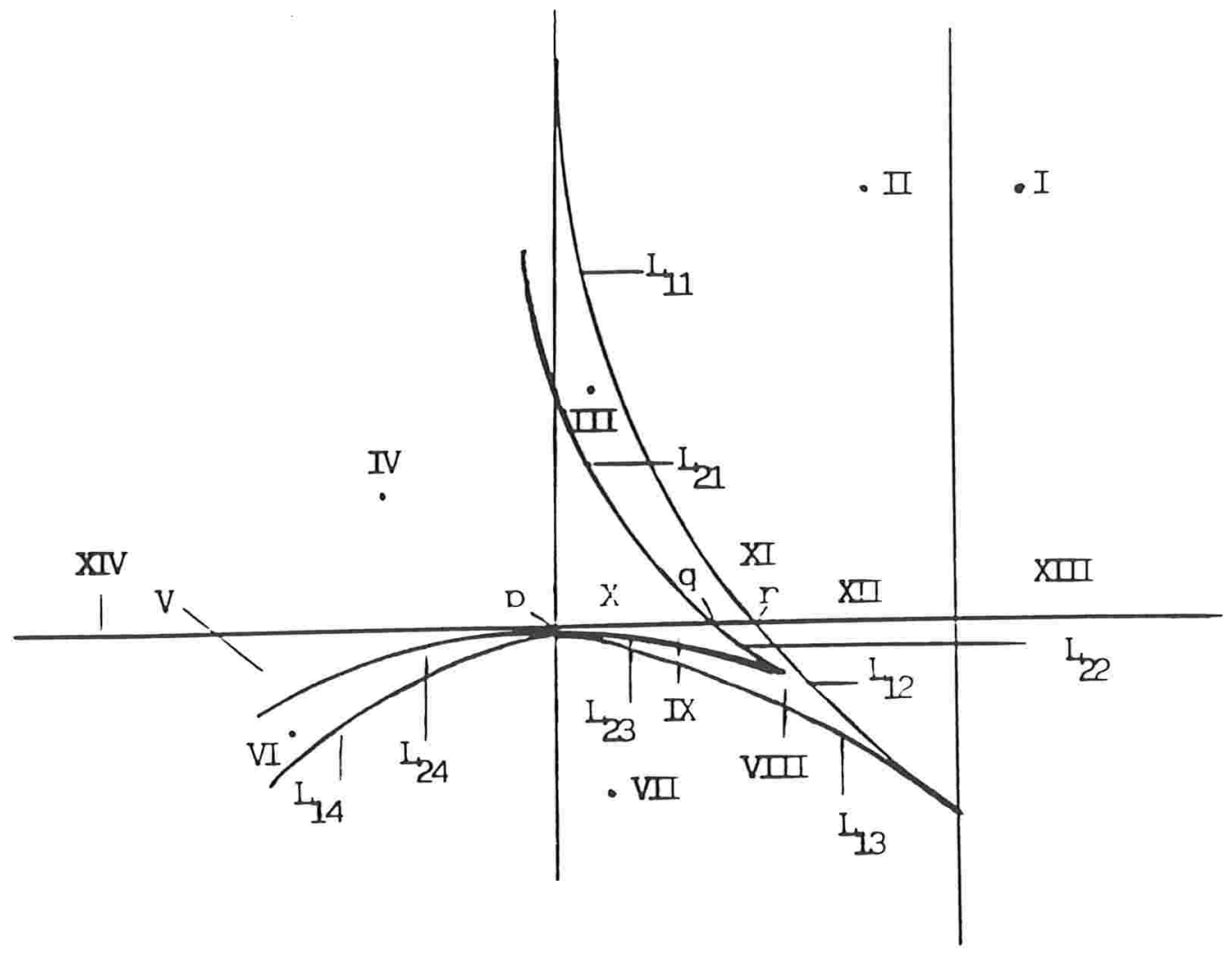

Figura 18: Cúspide que Definem o Número de Zeros e Pontos Críticos 


\begin{tabular}{|c|c|c|c|}
\hline REGIÃO & ZEROS & CRÍTICOS & NFFLEXÕES \\
\hline I & 2 & 1 & 0 \\
\hline II & 2 & 1 & 2 \\
\hline III & 2 & 3 & 2 \\
\hline$\Gamma \mathrm{V}$ & 4 & 3 & 2 \\
\hline V & 4 & 3 & 2 \\
\hline VI & 2 & 3 & 2 \\
\hline VII & 2 & 1 & 2 \\
\hline VIII & 2 & 3 & 2 \\
\hline LX & 4 & 3 & . 2 \\
\hline $\mathrm{X}$ & 3 & 3 & 2 \\
\hline $\mathrm{XI}$ & 1 & 3 & 3 \\
\hline XII & 1 & 1 & 2 \\
\hline XIII & 1 & 1 & 0 \\
\hline XIV & 3 & 3 & 2 \\
\hline L11 & 2 & 2 & 2 \\
\hline L12 & 2 & 2 & 2 \\
\hline L13 & 2 & 2 & 2 \\
\hline L14 & 2 & 2 & 2 \\
\hline L21 & 3 & 3 & 2 \\
\hline L22 & 3 & 3 & 2 \\
\hline L23 & 3 & 3 & 2 \\
\hline L24 & 3 & 3 & 2 \\
\hline $\mathrm{P}$ & 2 & 2 & 2 \\
\hline $\mathrm{Q}$ & 2 & 3 & 2 \\
\hline $\mathrm{R}$ & 1 & 2 & 2 \\
\hline
\end{tabular}

Tabela II: Número de Zeros.Pontos Críticos e Pontos de Inflexão de $F_{b . c}$ 


\section{3 - AS SINGULARIDADES DO SISTEMA $X_{s, b, c}:\left\{\begin{array}{c}x^{\prime}=y-\left(b x+c x^{2}-4 x^{3}+x^{4}\right) \\ y^{\prime}=s\left(x^{2}-2 x\right)\end{array}\right.$}

A singularidades do sistema $X_{\varepsilon, b . c}$. dado por (10). são os pontos $(0,0)$ e $\left(2, F_{b, c}(2)\right)$ se $\varepsilon \neq 0$. A matriz representando $j^{1} X_{\varepsilon, b, c}$ em $(0,0)$ é dada por:

$$
\left[\begin{array}{cc}
-b & 1 \\
-2 \varepsilon & 0
\end{array}\right]
$$

A equação dos auto-valores é dada por:

$$
z^{2}+b z+2 \varepsilon=0
$$

Se $b^{2}-8 \varepsilon<0$ temos um foco repulsor para $b<0$ e um foco atrator para $b>0$. Os autovalores admitem parte real igual a zero se e somente se $b=0$. Para $b^{2}-8 \varepsilon \geq 0$ temos um nó repulsor se $b<0$ e um nó atrator se $b>0$.

A matriz representando $j^{1} X_{\varepsilon, b, c}$ em $(2,2 b+4 c-16)$ é dada por:

$$
\left[\begin{array}{cc}
-(b+4 c-16) & 1 \\
2 \varepsilon & 0
\end{array}\right]
$$

A equação dos auto-valores é dada por:

$$
z^{2}+(b+4 c-16) z-2 \varepsilon=0
$$

Para $\varepsilon>0$ segue que a singularidade $(2,2 b+4 c-16)$ é um ponto de sela. A sela ocorre num ponto crítico do gráfico de $F_{b, c}$ para valores do parâmetro no plano dado por:

$$
(S C): b=-4 c+16
$$

Vamos agora analisar a singularidade $(0,0)$ quando $b=0$. 


\section{Teorema. 6}

Seja $X_{\varepsilon, b . c}$ dado por (10). Temos:

a) $(x, y)=(0,0)$ é um. foco atrator (ou nó) se b>0 e um foco repulsor (ou nó) se $b<0$.

b) $(x, y)=(0,0)$ é um foco atrator fraco se $b=0$ e $c \geq 12$ e um foco repulsor fraco se $b=0$ e $c<12$.

c) Para valores do parâmetro em $H=\{b=0, c \neq 12\}$ ocorre uma Bifurcação de Hopf de codimensão 1 , surgindo um ciclo atrator em $b<0$ e $c>12$ e um ciclo repulsor em $b>0$ e $c<12$.

d) Para valores do parâmetro em $H_{2}=\{b=0, c=12\}$ ocorre uma Bifurcação de Hopf de codimensão 2 surgindo 2 ciclos em $b>0$ e $c<12$. O ciclo interno será repulsor e o outro atrator.

\section{Demonstraçāo:}

A divergência de $X_{\varepsilon . b . c}$ é nula em $(0,0)$ se e somente se $b=0$. Tomando $b=0, X_{\varepsilon, b . c}$ assume a forma

$$
\left\{\begin{array}{l}
x^{\prime}=y-c x^{2}+4 x^{3}-x^{4} \\
y^{\prime}=\varepsilon\left(x^{2}-2 x\right)
\end{array}\right.
$$

Efetuando a mudança (28) e denotando $y=y_{1}$ obtemos (29)

$$
\begin{gathered}
y=\sqrt{2 \varepsilon} y_{1} \\
\left\{\begin{array}{l}
x^{\prime}=\sqrt{2 \varepsilon} y-c x^{2}+4 x^{3}-x^{4} \\
y^{\prime}=-\sqrt{2 \varepsilon} x+\frac{\sqrt{2 \varepsilon}}{2} x^{2}
\end{array}\right.
\end{gathered}
$$

Efetuamos a mudança (30): 


$$
\begin{aligned}
& x=r \cos \theta \\
& y=r \operatorname{sen} \theta
\end{aligned}
$$

Em coordenadas polares (27) é expresso por:

$$
\left\{\begin{array}{l}
r^{\prime}=\left(\frac{\sqrt{2 \varepsilon}}{2} \operatorname{sen} \theta-c \cos \theta\right) \cos ^{2} \theta r^{2}+4 \cos ^{4} \theta r^{3}-\cos ^{5} \theta r \\
\theta^{\prime}=-\sqrt{2 \varepsilon}+\left(\frac{\sqrt{2 \varepsilon}}{2} \cos \theta+c \operatorname{sen} \theta\right) \cos ^{2} \theta r-4 \cos ^{3} \theta \operatorname{sen} \theta r^{2}+\cos ^{4} \theta \operatorname{sen} \theta r^{3}
\end{array}\right.
$$

O retrato de fase de (31) corresponde aos gráficos das funções $r=f_{\rho}(\theta)$, $f_{\rho}(0)=\rho$, soluções de:

$$
\begin{gathered}
R(r, \theta)=\frac{d r}{d \theta}=R_{1}(\theta) r+R_{2}(\theta) r^{2}+\ldots \\
R_{k}(\theta)=\left.\frac{1}{k !} \frac{\partial^{k} R(r, \theta)}{\partial r^{k}}\right|_{r=0}
\end{gathered}
$$

Desenvolvendo $f \rho(\theta)$ em potências de $\rho$ obtemos:

$$
f_{\rho}(\theta)=u_{1}(\theta) \rho+u_{2}(\theta) \rho^{2}+\ldots
$$

Substituindo $r=f_{\rho}(\theta)$ em (32) obtemos:

$$
u_{1}^{\prime}(\theta) \rho+u_{2}^{\prime}(\theta) \rho^{2}+\ldots=R_{1}(\theta)\left[u_{1}(\theta) \rho+\ldots\right]+R_{2}(\theta)\left[u_{1}(\theta) \rho+\ldots\right]^{2}+\ldots
$$

Assim, conforme $[A L G M]$, os valores de $u_{i}(\theta)$ são obtidos recursivamente por: 


$$
\begin{aligned}
& u_{1}(\theta)=1 \\
& u_{2}(\theta)=\int R_{2}(s) d s \\
& u_{3}(\theta)=\int\left(2 R_{2}(s) u_{2}(s)+R_{3}(s)\right) d s \\
& u_{4}(\theta)=\int\left[2 R_{2}(s) u_{3}(s)+R_{2}(s) u_{2}^{2}(s)+3 R_{3}(s) u_{2}(s)+R_{4}(s)\right] d s \\
& u_{5}(\theta)=\int\left[2 R_{2}(s)\left(u_{4}(s)+u_{2}(s) u_{3}(s)\right)+3 R_{3}(s)\left(u_{3}(s)+u_{2}^{2}(s)\right)+4 R_{4}(s) u_{2}(s)\right] d s
\end{aligned}
$$

A aplicação de retorno $\pi: R^{+} \rightarrow R$ é definida por:

$$
\pi(\rho)=f_{\rho}(2 \pi)-\rho=u_{2}(2 \pi) \rho^{2}+\ldots
$$

Os coeficientes de Lyapunov são dados por:

$$
V_{k}=\frac{\pi^{(k)}(0)}{k !}
$$

Para o cálculo dos coeficientes utilizamos o MAPLE e obtemos os seguintes valores:

$$
\begin{aligned}
& V_{1}=0 \\
& V_{2}=0 \\
& V_{3}=\frac{\pi(c-12)}{4 \sqrt{2 \varepsilon}} \\
& V_{4}=0 \\
& V_{5}=\frac{\sqrt{2} \pi}{1024(\sqrt{2 \varepsilon})^{3}}\left(-168 c^{2}+21 \varepsilon c-1152 \sqrt{2 \varepsilon} \pi c+6912 \sqrt{2 \varepsilon} \pi-76 \varepsilon+14 c^{3}+48 \sqrt{2 \varepsilon} \pi c^{2}\right)
\end{aligned}
$$

Quando $b=0$ temos $V_{1}=V_{2}=0$ e $V_{3}=0 \Longleftrightarrow c=12$. Assim quando $c>12$ temos que $V_{3}>0$

O sentido da rotação é o sentido horário. Assim para $V_{3}>0$ temos um foco fraco atrator e para $V_{3}<0$ um foco fraco repulsor. 

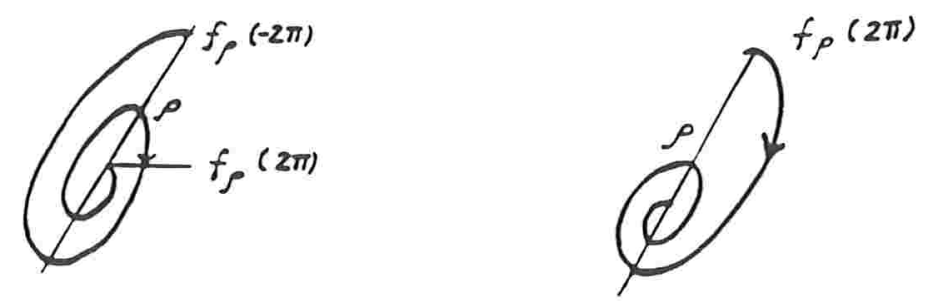

Figura 19:A Aplicação de Retorno $\pi$

Quando $c=12$ temos:

$$
V_{5}=\frac{11}{32} \frac{\pi}{\sqrt{2 \varepsilon}}
$$

Como $V_{5}$ é positivo segue que $(0,0)$ é um foco fraco atrator. Observemos também que quando $\varepsilon \downarrow 0$ temos $V_{5} \rightarrow+\infty$. 


\section{4 - OS RETRATOS DE FASE DO SISTEMA $\mathrm{X}_{\text {s.b. }}$}

Inicialmente vamos analisar o retratu de fase de $X_{s, b, r}$ dado por $(10)$ quandu $\varepsilon=0$. Neste caso o sistema (10) assume a forma

$$
\left\{\begin{array}{l}
x^{\prime}=!-F_{b . c}(x) \\
y^{\prime}=0
\end{array}\right.
$$

Para cada par de valores fixados $b$ e $c$ temos que as singularidades de (41) são os pontos $(x, y)$ tais que $y=F_{b, c}(x)$ ( ou seja na curva $L_{b . c}$ dada por 12). Excluindo os pontos críticos, todas as singularidades são normalmente hiperbólicas. A figura 23 mostra o retrato de fase para um valor fixo $(b, c)$.

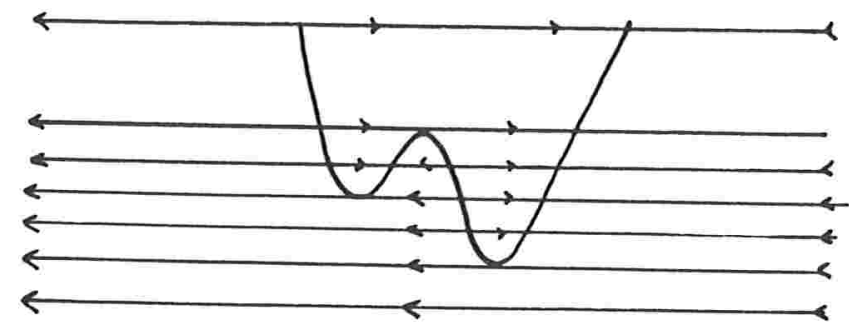

Figura 20: Retratos de Fase de $X_{0 . b . c}$

Vamos agora analisar quando $\varepsilon \neq 0$. Quando as retas $x=0$ e $x=2$ cruzam transversalmente o gráfico de $y=F_{b, c}(x)$ em pontos não críticos podemos utilizar o lema de R. Lutz e M. Goze [LG] para esboçar os retratos de fase. As trajetórias são tais que

$$
\frac{d y}{d x}=\frac{\varepsilon\left(x^{2}-2 x\right)}{y-F_{b . c}(x)}
$$

Assim o campo de vetores é "quase" horizontal quando $\varepsilon \downarrow 0$. Como vimos em 3 . em $b=0$ nasce um ciclo limite (ou 2 ciclos limites, quando $c=12$ ). 


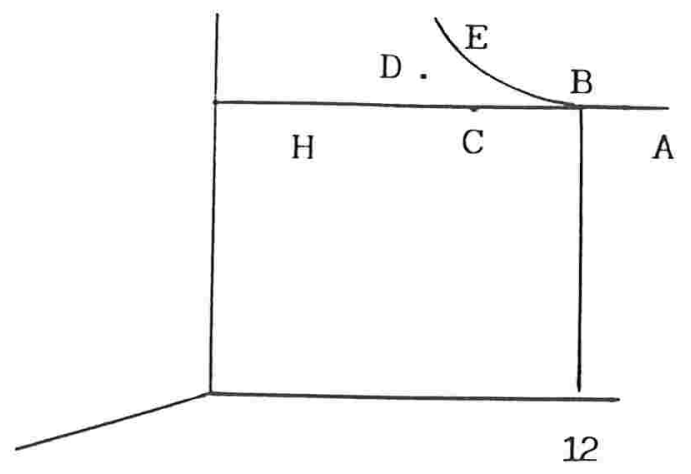

Figura 21: Alguns Pontos do Espaço $(\varepsilon, b, c)$

Assim para $\varepsilon \downarrow 0$ e $b \rightarrow 0$ uma parte do retrato de fase é dada na figura 22

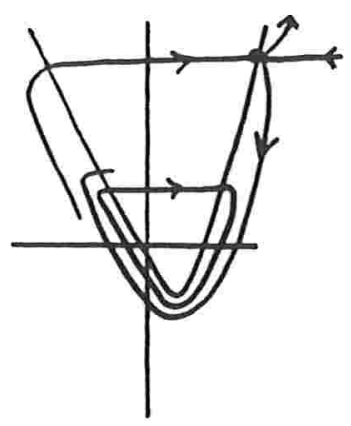

A

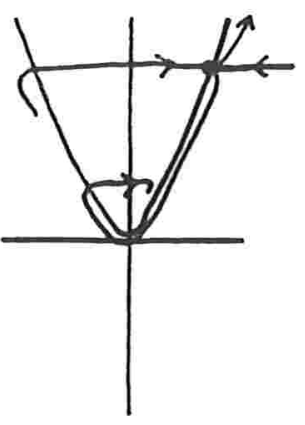

B

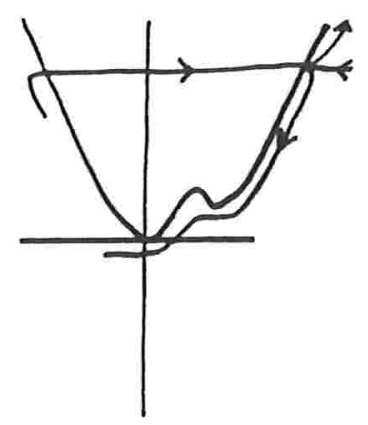

c

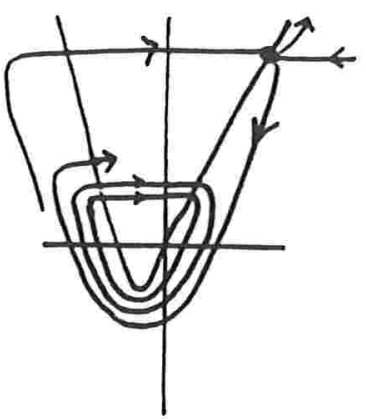

D

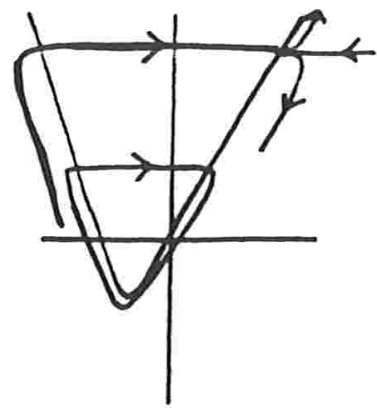

$E$

Figura 22 :Retratos de Fase em A, B. C.D e E.. 
Surgem duas dificuldades para obtenção total do retrato de fase:

1) O número de ciclos que o sistema admite.

2) As posições das separatrizes de sela.

Consideremos o plano dado por (26), isto é. valores do parâmetro para os quais o ponto de sela ocorre num ponto crítico.

Quando $b>-4 c+16$ o ponto de sela está à direita do mínimo local e quando $b<-4 c+16$ está a esquerda do mínimo local.

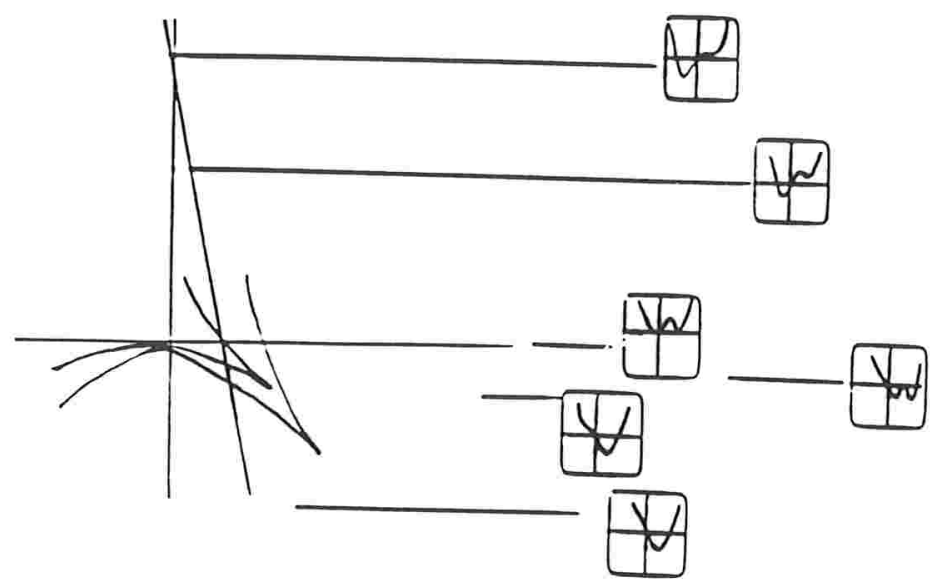

Figura 23: Gráficos de $F_{b, c}$ no plano (SC) 
Consideremos também o plano dado por (43). Para valores do parâmetro em (43) temos $F_{b, c}(0)=F_{b, c}(2)$.

$$
\left(S C_{2}^{\prime}\right) b=-2 c+8
$$
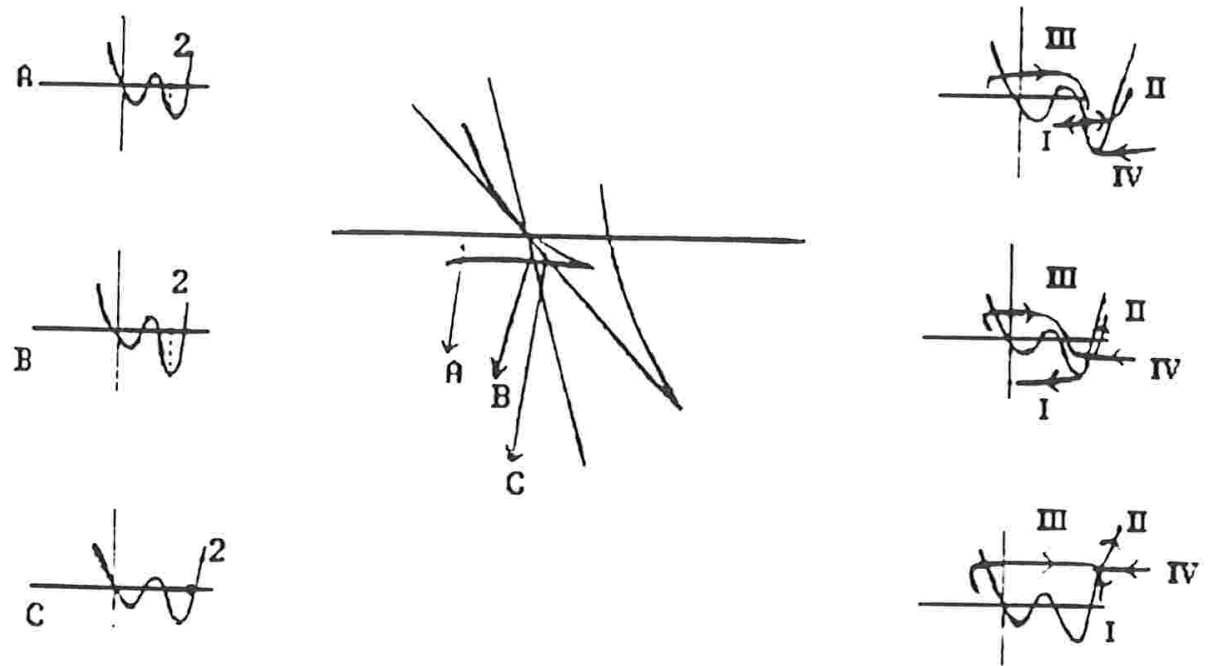

Figura 24: Planos $\mathrm{SC}$ e $\mathrm{SC}_{2}$ 
Se $\varepsilon \downarrow 0$, I e II assumem as posições indicadas na figura 23 pois o campo de vetores é quase horizontal. III e IV estão numa vizinhança do gráfico de $y=F_{b, c}(x)$ (conhecido por Variedade Lenta) e as orientações são conseqüências das orientações de I e II. Quando II cruza o gráfico de $y=F_{b, c}(x)$ não pode descer pois se isto ocorresse teríamos uma terceira singularidade. A mesma razão justifica III.

Este comportamento é o mesmo até atingirmos o plano dado por (26).

A mudança nas posições relativas das separatrizes de sela : para os parâmetros em (26) , poderá ocorrer quando a separatriz instável deixar a variedade lenta num ponto com a mesma altura que o ponto de mínimo. Assim, para $\varepsilon \downarrow 0$, o limite dos pontos que satisfazem está condição é $(b, c)=(0,4)$.

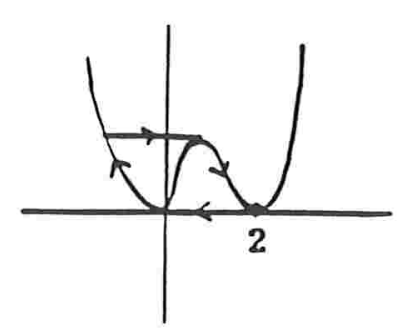

Figura 25:Limite das Órbitas Homoclínicas de Codimensão 2 quando $\varepsilon \downarrow 0$

Com relação ao número de ciclos vamos utilizar o seguinte lema (Teorema de Copel) que encontra-se em [DR2]. 
Lema 7 Consideremos o sistema (44):

$$
\left\{\begin{array}{l}
x^{\prime}=F(x)-y \\
y^{\prime}=g(x)
\end{array}\right.
$$

Suponhamos que $F$ é de classe $C^{2}$ e que $g$ é de classe $C^{1}$. Além disso supomos $F$ e g. definidas para $x \in(\alpha, \beta)$, satisfazendo:

1) $f(x)=F^{\prime}(x)$ admite um único zero $x_{0}<0, f(x)<0(>0)$ quando $\alpha<x<x_{0}$ $\left(x_{0}<x<\beta\right)$.

2) $F(0)=0, F\left(\xi_{0}\right)=0$ para $\alpha<\xi_{0}<x_{0}$.

3) $x g(x)>0$ para $x \neq 0, x \in(\alpha, \beta)$.

Então não existe ciclo limite na região $\xi_{0}<x<\beta$.
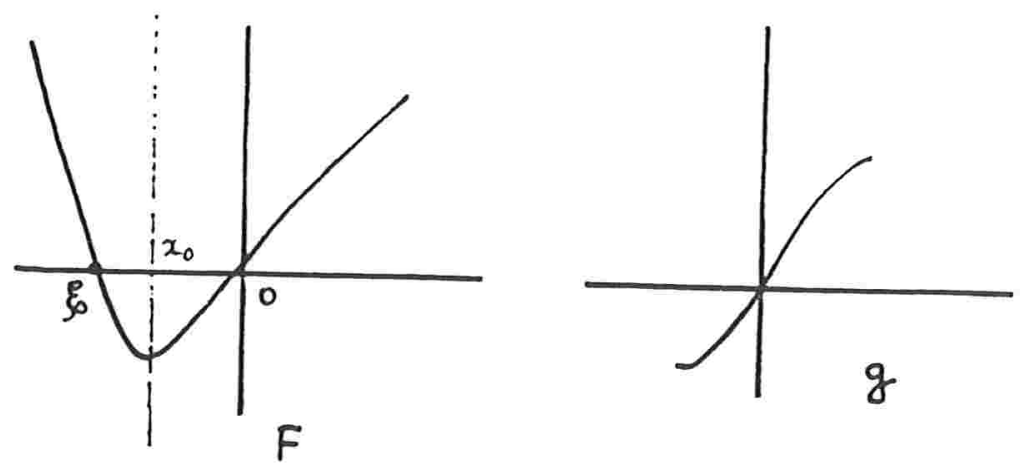

Figura 26: As funções F e $g$ do Lema 7

A prova do Lema de Copel baseia-se numa simetria dada pela reta onde a divergência anula-se. 
Consideremos $b \neq 0$ e $c \in \mathbb{R}$ fixos e suponhamos que $X_{\varepsilon, b, c}$. dado por (10). admite um ciclo limite $\Gamma_{\varepsilon}$. Quando $\varepsilon \downarrow 0$ ou este ciclo desaparece ou ele terá como LCP o ponto (0.0). De fato. se $b \neq 0$ a singularidade $(0.0)$ não ocorre num ponto crítico.

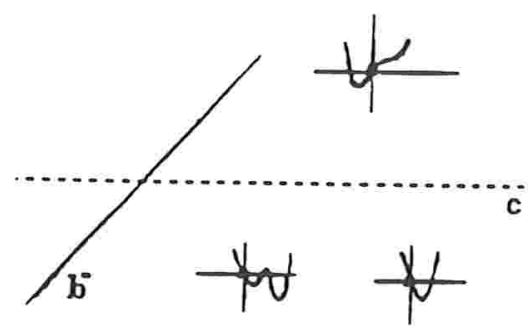

Figura 27: LCP's para $b \neq 0$

Teorema. 8 Seja $X_{s, b, c}$ dado por (10) com $b \neq 0$. Então $\exists \varepsilon_{0}>0, \varepsilon_{0}=\varepsilon_{0}(b, c)$, tal que se $0<\varepsilon<\varepsilon_{0}$ então $X_{\text {s.b.c }}$ não admite ciclos limites.

\section{Demonstraçāo:}

Suponhamos que dados $b, c \in \mathbb{R}, b \neq 0, \exists \varepsilon_{n} \rightarrow 0$ tal que $X_{\varepsilon_{n}, b, c}$ admite um ciclo limite $\Gamma_{s n}$. Pela observação anterior segue $\Gamma_{\varepsilon_{n}} \rightarrow\{(0,0)\}$.

Se $b>0$ então $-X_{\varepsilon_{n}, b, c}$ satisfaz as hipóteses 1,2 e 3 do lema 7 , para uma conveniente escolha de $(\alpha, \beta)$. Logo $X_{\varepsilon_{n}, b . c}$ não admite ciclos numa vizinhança de $\{(0,0)\}$.

Se $b<0$ então consideremos a substituição $x_{1}=-x, y_{1}=y$. Temos que

$$
Y_{\varepsilon_{n} b, c}:\left\{\begin{aligned}
x_{1}^{\prime} & =\tilde{F}_{b, c}\left(x_{1}\right)-y_{1} \\
y_{1}^{\prime} & =\varepsilon\left(x_{1}^{2}+2 x_{1}\right)
\end{aligned}\right.
$$

satisfaz as hipóteses do lema 6 e portanto $Y_{s_{n}, b . c}$ não admite ciclos numa vizinhança de $\{(0,0)\}$.Estamos considerando $\widetilde{F}_{b, c}\left(x_{1}\right)=F_{b, c}\left(-x_{1}\right)$. 


\section{5 - A DESINGULARIZAÇĀO DE $X_{\varepsilon, b . c}$}

5-1-A desingularizaçāo em $(x, y, \varepsilon, b, c)=(0.0 .0 .0 .12)$

Como vimos em $3,(\varepsilon, b, c)=(0,0,12)$ é o limite dos pontos onde ocorre a Bifurcação de Hopf de codimensão 2. Além disso $(x, y)=(0,0)$ não é uma singularidade normalmente hiperbólica quando $(\varepsilon, b, c)=(0,0,12)$. Para simplificar os cálculos vamos transladar $c=12$ para $c=0$. Começamos com a mudança (46)

$$
c_{1}=c-12
$$

Se denotamos $c=c_{1}$ obtemos

$$
X_{s . b . c}:\left\{\begin{array}{l}
x^{\prime}=y-b x-c x^{2}-12 x^{2}+4 x^{3}-x^{4} \\
y^{\prime}=\varepsilon\left(x^{2}-2 x\right)
\end{array}\right.
$$

Quando $\varepsilon=b=c=0$ o retrato de fase é dado por

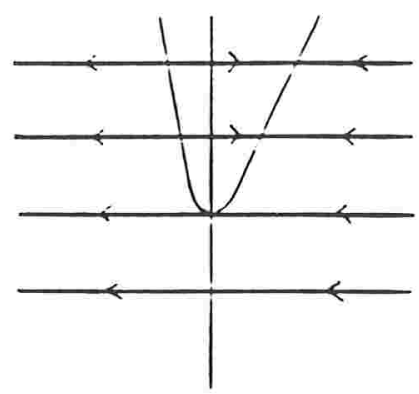

Figura 28: Retrato de Fase Quando $(\varepsilon, b, c)=(0,0,0)$

O ponto $(x, y)=(2,32)$ é o limite dos pontos de sela quando $(\varepsilon, b, c) \rightarrow(0,0,0)$. Para $(\varepsilon, b, c)$ numa vizinhança de $(0,0,0), X_{\varepsilon, b . c}$ admite até dois ciclos limites. Nosso objetivo é estudar o que ocorre com estes ciclos quando $(\varepsilon, b, c) \rightarrow(0,0,0)$.

Consideremos a aplicação $\varphi:[0, T] \times S_{+}^{4} \rightarrow \mathbb{R}^{5}$ dada por

$$
\begin{array}{ll}
x=u \bar{x} & \varepsilon=u^{2} \bar{\varepsilon} \\
y=u^{2} \bar{y} & b=u \bar{b} \\
& c=u \bar{c}
\end{array}
$$


com $u \in[0, T],(\bar{x}, \bar{y}, \bar{\varepsilon}, \bar{b}, \bar{c}) \in S_{+}^{4}$ e $T>0$.

Temos que existe $\widehat{X}$ em $[0, T] \times S_{+}^{4}$ tal que $\varphi_{*}(\widehat{X})=X_{\varepsilon, b, c}$. Dividindo $\widehat{X}$ por $u$ o campo de vetores resultante $\bar{X}$ será chamado de campo de vetores desingularizado em $[0, T] \times S_{+}^{4}$.

$$
\bar{X}:\left\{\begin{array}{l}
\bar{x}^{\prime}=\bar{y}-\bar{b} \bar{x}-u \overline{c x}^{2}-12 \bar{x}^{2}+4 u \bar{x}^{3}-u^{2} \bar{x}^{4} \\
\bar{y}^{\prime}=\bar{\varepsilon}\left(u \bar{x}^{2}-2 \bar{x}\right)
\end{array}\right.
$$

Estamos considerando $S^{4}=\left(S^{2} \times D\right) \cup\left(D^{2} \times S^{1}\right)$ onde $S^{2}=\left\{(\bar{\varepsilon}, \bar{b}, \bar{c}) \mid \bar{\varepsilon}^{2}+\bar{b}^{2}+\bar{c}^{2}=1\right\}$, $D=\{(\bar{x}, \bar{y}) \mid \bar{x} \in[0,1], \bar{y} \in[0,1]\}, D^{2}=\left\{(\bar{\varepsilon}, \bar{b}, \bar{c}) \mid \bar{\varepsilon}^{2}+\bar{b}^{2}+\bar{c}^{2}<1, \bar{\varepsilon}>0\right\}$ e $S^{1}=\{(\bar{x}, \bar{y}) \mid$ $\left.\bar{x}^{2}+\bar{y}^{2}=1\right\}$.

Seja $M_{0}=\{(x, y)\} \times\{(\varepsilon, b, c)\}$ e $T_{1}=[0, T] \times S_{+}^{4}$.

Definimos $M_{1}=\left(T_{1} \cup M_{0}-\{0\}\right) / m \sim \varphi(m)$.

Sejam $\phi: M_{1} \rightarrow M_{0}$ tal que $\phi \mid\left(S^{4} \times\{0\}\right)=\varphi$ e $\phi \mid M_{0}-\{0\}=i d$

Definimos $\pi: M_{0} \rightarrow\{(\varepsilon, b, c)\}$ por $\pi(x, y, \varepsilon, b, c)=(\varepsilon, b, c)$ e $\widehat{\pi}: M_{1} \rightarrow\{(\varepsilon, b, c)\}$ por $\widehat{\pi}(x, y, \varepsilon, b, c)=\pi(\phi(x, y, \varepsilon, b, c))$.

A fibra singular $F_{0}$ corresponde a $\widehat{\pi}^{-1}(0,0,0)$ e equivale a $\left(S^{4} \times\{0\}\right) \cup\left(S^{1} \times \mathbb{R}\right)$.

Sejam $\bar{\lambda}_{0}=\left(\varepsilon_{0}, b_{0}, c_{0}\right) \in S^{2}, l_{\bar{\lambda}_{0}}=\left\{t \bar{\lambda}_{0} \mid t \in \mathbb{R}\right\}$ e $P_{\bar{\lambda}_{0}}=\widehat{\pi}^{-1}\left(l_{\bar{\lambda}_{0}}\right)$. Temos que $P_{\bar{\lambda}_{0}}$ é um espaço tridimensional e que $\lambda$ define uma fibra regular $F_{\lambda}$ quando $\lambda \neq 0$. 


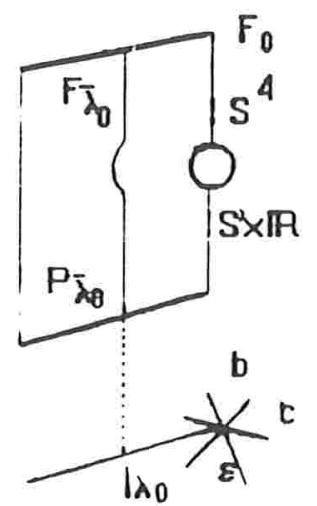

Fìgura 29: Folheação de $M_{1}$

Definimos $\tilde{X}$ em $M_{1}$ por

$$
\tilde{X}:\left\{\begin{array}{l}
X_{\varepsilon, b, c} \text { em } M_{1}-\{0\} \\
\bar{X} \text { em } T_{1}
\end{array}\right.
$$

Em $S^{1} \times \mathbb{R}$ temos $\bar{\varepsilon}=\bar{b}=\bar{c}=0$. Efetuamos a mudança (51) e obtemos (52) onde $c=\cos \theta$ e $s=\operatorname{sen} \theta$.

$$
\begin{gathered}
x=u \cos \theta \\
y=u^{2} \operatorname{sen} \theta \\
\left\{\begin{array}{l}
u^{\prime}=\frac{u c}{c^{2}+2 s^{2}}\left(s-12 c^{2}+4 u c^{2}-u^{2} c^{4}\right) \\
\theta^{\prime}=\frac{-2 s}{c^{2}+2 s^{2}}\left(s-12 c^{2}+4 u c^{2}-u^{2} c^{4}\right)
\end{array}\right.
\end{gathered}
$$

As singularidades de $(52)$ são $(0,0),(\pi, 0),\left(\theta_{1}, 0\right)$ e $\left(\theta_{2}, 0\right)$ onde $\theta_{1}$ e $\theta_{2}$ são soluções de $s=12 c^{2}$. Temos que $\theta_{1} \in\left(0, \frac{\pi}{2}\right)$ e que $\theta_{2} \in\left(\frac{\pi}{2}, \pi\right)$. Multiplicando (52) por $\left(c^{2}+2 s^{2}\right)$ obtemos

$$
\left\{\begin{array}{c}
\theta^{\prime}=-2 s\left(s-12 c^{2}+4 u c^{2}-u^{2} c^{4}\right) \\
u^{\prime}=u c\left(s-12 c^{2}+4 u c^{2}-u^{2} c^{4}\right)
\end{array}\right.
$$

Assim obtemos:

$$
D X(\theta, 0)=\left[\begin{array}{cc}
-4 s c+24 c^{3}-48 s^{2} c & -8 c^{2} s \\
0 & s c-12 c^{3}
\end{array}\right]
$$




$$
\begin{aligned}
& D X(0,0)=\left[\begin{array}{cc}
24 & 0 \\
0 & -12
\end{array}\right] \\
& D X(\pi, 0)=\left[\begin{array}{cc}
-24 & 0 \\
0 & 12
\end{array}\right]
\end{aligned}
$$

Em $(\theta, u)=(0,0)$ temos que o auto-valor angular é positivo e o auto-valor radial é negativo. Em $(\theta, u)=(\pi, 0)$ temos auto-valor angular negativo e auto-valor radial positivo.

Se $\theta=\theta_{1}$ ou $\theta=\theta_{2}$ temos $-4 s c+24 c^{3}-48 s^{2} c=-2 s c-48 s^{2} c<0$ para $\theta=\theta_{1} \mathrm{e}$ $-2 s c-48 s^{2} c>0$ para $\theta=\theta_{2}$. Além disso os auto-valores são nulos na parte radial.

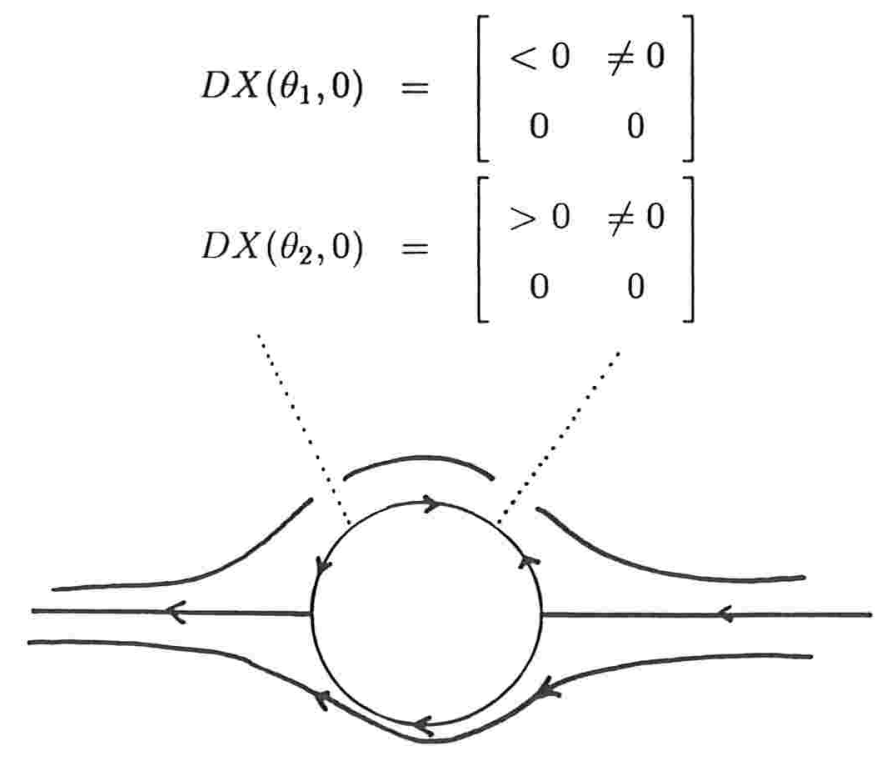

Figura 30: Retrato de Fase de $\tilde{X}$ em $F_{0} \mid S^{1} \times R$

Vamos fixar $\bar{\varepsilon}=1$. Tomando $u=0$ em (49) obtemos

$$
\bar{X}_{\bar{b} . \bar{c}}:\left\{\begin{array}{l}
\bar{x}^{\prime}=\bar{y}-\bar{b} \bar{x}-12 \bar{x}^{2} \\
\bar{y}^{\prime}=-2 \bar{x}
\end{array}\right.
$$

Temos que $\bar{X}_{\bar{b}, \bar{c}}$ independe do valor de $\bar{c}$ e quando $\bar{b}=0$ (49) apresenta um centro simétrico $\mathrm{em}(\bar{x}, \bar{y})=(0,0)$

$$
\bar{X}_{0}:\left\{\begin{array}{c}
\bar{x}^{\prime}=\bar{y}-12 \bar{x}^{2} \\
\bar{y}^{\prime}=-2 \bar{x}
\end{array}\right.
$$


O retrato de fase de $\tilde{X}$ em $P_{(1,0, \bar{c})}$ quando $u=0$ é dado por

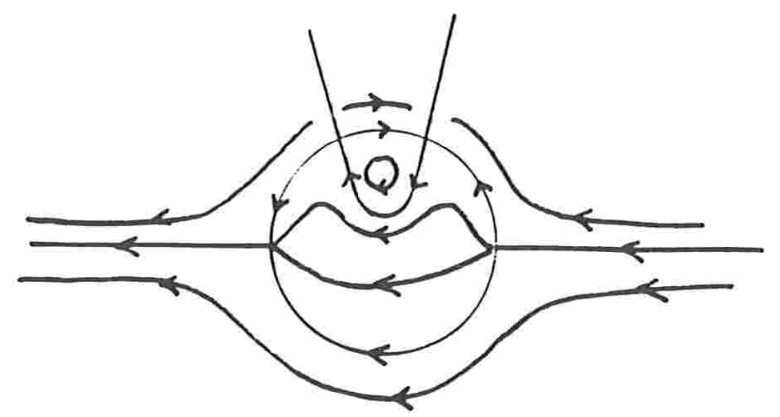

Figura 31 : Retrato de Fase de $\tilde{X}$ quando $\bar{\varepsilon}=1, \bar{b}=0$ e $u=0$

Se tomamos $\bar{\varepsilon}=1, \bar{b}=\bar{b}_{0}>0, u=0$ ou $\bar{\varepsilon}=1, \bar{b}=\bar{b}_{1}<0, u=0$ os retratos estão representados na figura
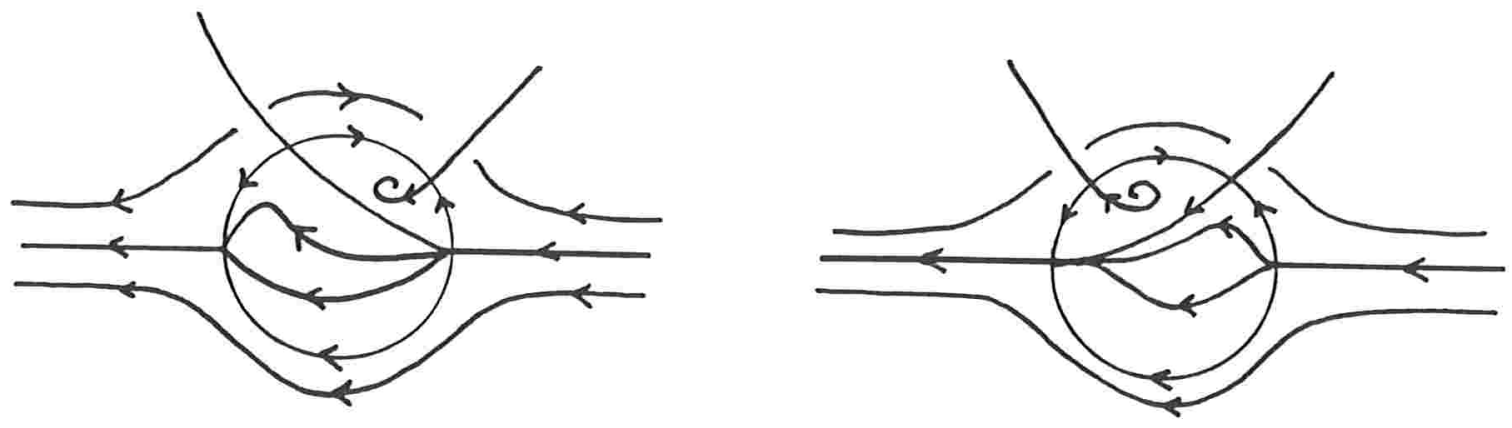

Figura 32 :Retratos de Fase de $\tilde{X}$ Quando $\bar{b}>0$ ou $\bar{b}<0$

5.2-A desingularizaçāo em $(x, y, \varepsilon, b, c)=(2,0,0,0,4)$

Iniciamos com a seguinte mudança:

$$
\begin{array}{ll}
x_{1}=x-2 & b_{1}=-b-4 c+16 \\
y_{1}=y+2 b-4 c+16 & c_{1}=c-4
\end{array}
$$

A mudança (58) transforma (10), voltando a chamar $x, y, b, c$,em:

$$
\left\{\begin{array}{l}
x^{\prime}=y+b x-c x^{2}-4 x^{2}-4 x^{3}-x^{4} \\
y^{\prime}=\varepsilon\left(x^{2}+2 x\right)
\end{array}\right.
$$


Vamos efetuar a desingularização no ponto $(0,0,0,0,0)$.

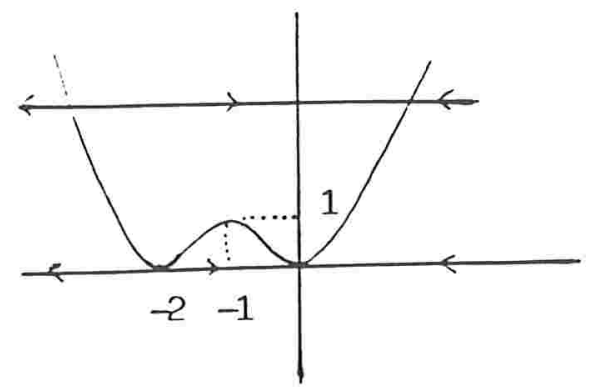

Figura 33:Retrato de Fase de $X_{0,0,4}$

Consideremos a aplicação $\Psi:[0, T] \times S_{+}^{4} \rightarrow R^{5}$ dada por:

$$
\begin{array}{rlrl}
x=u \bar{x} & b & =u \bar{b} \\
y=u^{2} \bar{y} & c & =u \bar{c} \\
\varepsilon & =u^{2} \bar{\varepsilon}
\end{array}
$$

Como antes, fazendo $\bar{X}=\frac{1}{u} \hat{X}$ e tomando $\bar{\varepsilon}=1$ obtemos:

$$
\left\{\begin{array}{l}
\bar{x}^{\prime}=\bar{y}+\bar{b} \bar{x}-4 \bar{x}^{2}-4 u \bar{x}^{3}-u^{2} \bar{x}^{4} \\
\bar{y}^{\prime}=2 \bar{x}+u \bar{x}^{2}
\end{array}\right.
$$

Quando $\bar{b}=\bar{c}=u=0$ obtemos:

$$
\left\{\begin{array}{l}
\bar{x}^{\prime}=\bar{y}-4 \bar{x}^{2} \\
\bar{y}^{\prime}=2 \bar{x}
\end{array}\right.
$$

A singularidade $(0,0)$ de $(62)$ é tal que

$$
D X(0,0)=\left[\begin{array}{ll}
0 & 1 \\
2 & 0
\end{array}\right]
$$


O polinômio característico é

$$
z^{2}-2=0
$$

Assim $(0,0)$ é um ponto de sela.

Consideremos a mudança

$$
\begin{aligned}
& x=u \cos \theta \\
& y=u^{2} \operatorname{sen} \theta
\end{aligned}
$$

Tomando $\varepsilon=b=c=0$ em (59) e chamando $s=\operatorname{sen} \theta$ e $c=\cos \theta$ obtemos:

$$
\left\{\begin{array}{l}
\theta^{\prime}=\frac{-2 u s}{c^{2}+2^{2}}\left(s-4 c^{2}-4 u c^{3}-u^{2} c^{4}\right) \\
u^{\prime}=\frac{u^{2} c}{c^{2}+2 s^{2}}\left(s-4 c^{2}-4 u c^{3}-u^{2} c^{4}\right)
\end{array}\right.
$$

Multiplicando por $\left(c^{2}+2 s^{2}\right)$ obtemos

$$
\left\{\begin{array}{l}
\theta^{\prime}=-2 s\left(s-4 c^{2}-4 u c^{3}-u^{2} c^{4}\right) \\
u^{\prime}=u c\left(s-4 c^{2}-4 u c^{3}-u^{2} c^{4}\right)
\end{array}\right.
$$

As singularidades de (67), quando $u=0$, são dadas por $(0,0),(\pi, 0),\left(0, \varphi_{1}\right)$ e $\left(0, \varphi_{2}\right)$ onde $\varphi_{1} e \varphi_{2}$ são soluções de $\operatorname{sen} \varphi=4 \cos ^{2} \varphi$.

Temos que:

$$
D X(\theta, u)=\left[\begin{array}{ll}
-4 s c-16 s^{2} c+8 c^{3} & 8 s c^{3} \\
0 & s c-4 c^{3}
\end{array}\right]
$$

Assim obtemos

$$
\begin{aligned}
& D X(\pi, 0)=\left[\begin{array}{ll}
-8 & 0 \\
0 & 4
\end{array}\right] \\
& D X(0,0)=\left[\begin{array}{cc}
8 & 0 \\
0 & -4
\end{array}\right]
\end{aligned}
$$

Isto implica que a singularidade $(\pi, 0)$ apresenta auto-valor angular negativo e auto-valor radial positivo e a singularidade (0.0) apresenta auto-valor angular positivo e radial negativo.

Também temos que $s c-4 c^{3}=-8 c^{3}-16 s^{2} c$ quando $\theta=\varphi_{1}$ ou $\theta=\varphi_{2}$. Assim $s c-4 c^{3}$ é 
negativo para $\theta=\varphi_{1}$ e positivo para $\theta=\varphi_{2}$. Assim temos:

$$
D X\left(\varphi_{1}, 0\right)=\left[\begin{array}{ll}
<0 & \neq 0 \\
0 & 0 \\
>0 & \neq 0 \\
0 & 0
\end{array}\right]
$$

$\operatorname{Em}\left(\varphi_{1}, 0\right)$ temos auto-valor angular negativo e auto-valor radial nulo. Em $\left(\varphi_{2}, 0\right)$ temos auto-valor angular positivo e radial nulo.

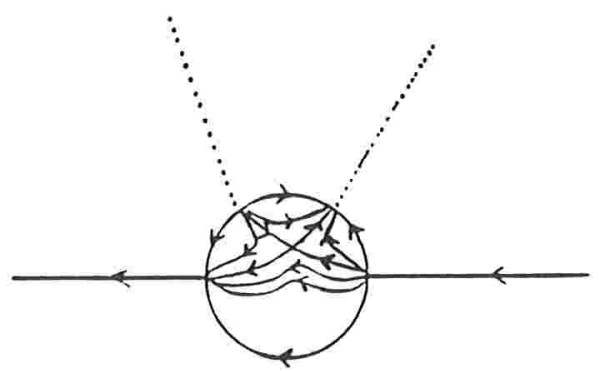

.Figura 34:Desingularização em. (2,0,0,0,4)

5.3-A desingularizaçāo em $(x, y, \varepsilon, b, c)=(1,1,0,0,4)$

Iniciamos com a seguinte mudança:

$$
\begin{array}{ll}
x_{1}=x-1 & b_{1}=-b-2 c+8 \\
y_{1}=y-b-c+3 & c_{1=c}-4
\end{array}
$$

A mudança (71) transforma (10), voltando a chamar $x, y, b, c$ em

$$
\left\{\begin{array}{l}
x^{\prime}=y+b x-c x^{2}+2 x^{2}-x^{4} \\
y^{\prime}=\varepsilon\left(x^{2}-1\right)
\end{array}\right.
$$

Vamos efetuar a desingularização no ponto $(0,0,0,0,0)$. 


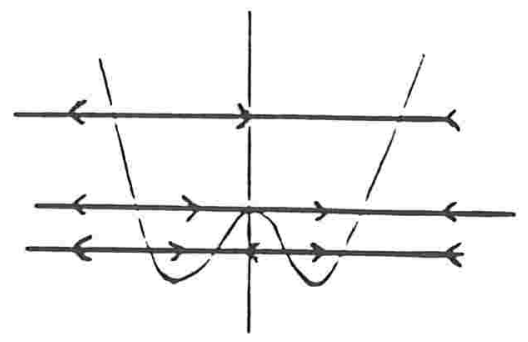

Figura 35:Retrato de Fase de $X_{\mathbf{0}, 0,4}$

Consideremos a aplicação $\delta:[0, T] \times S_{+}^{4} \rightarrow R^{5}$ dada por

$$
\begin{array}{rlrl}
x=u \bar{x} & b & =u \bar{b} \\
y=u^{2} \bar{y} & c & =u \bar{c} \\
& \varepsilon=u^{3} \bar{\varepsilon}
\end{array}
$$

Como antes, fazendo $\bar{X}=\frac{1}{u} X$ e tomando $\bar{\varepsilon}=1$ obtemos

$$
\left\{\begin{array}{l}
\bar{x}^{\prime}=\bar{y}+\bar{b} \bar{x}-u \overline{c x}^{2}+2 \bar{x}^{2}-u^{2} \bar{x}^{4} \\
\bar{y}^{\prime}=\left(u^{2} \bar{x}^{2}-1\right)
\end{array}\right.
$$

Quando $\bar{b}=\bar{c}=u=0$ obtemos

$$
\left\{\begin{array}{l}
\bar{x}^{\prime}=\bar{y}+2 \bar{x}^{2} \\
\bar{y}^{\prime}=-1
\end{array}\right.
$$

O campo de vetores (75) não admite singularidades e seu retrato de fase é dado por 


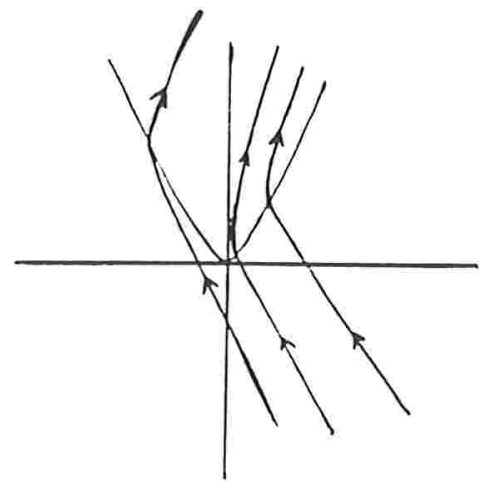

Figura 36:Retrato de Fase de (75)

\section{Consideremos a mudança}

$$
\begin{aligned}
& x=u \cos \theta \\
& y=u \operatorname{sen} \theta
\end{aligned}
$$

Tomando $\varepsilon=b=c=0$ em (72) e denotando $s=\operatorname{sen} \theta$ e $c=\cos \theta$ obtemos

$$
\left\{\begin{array}{l}
\theta^{\prime}=\frac{-2 s}{\left(c^{2}+2 s^{2}\right)}\left(s+2 c^{2}-u^{2} c^{4}\right) \\
u^{\prime}=\frac{u c}{\left(c^{2}+2 s^{2}\right)}\left(s+2 c^{2}-u^{2} c^{4}\right)
\end{array}\right.
$$

Multiplicando por $c^{2}+2 s^{2}$ obtemos

$$
\left\{\begin{array}{l}
\theta^{\prime}=-2 s\left(s+2 c^{2}-u^{2} c^{4}\right) \\
u^{\prime}=u c\left(s+2 c^{2}-u^{2} c^{4}\right)
\end{array}\right.
$$

As singularidades de (78), quando $u=0$, são dadas por $(0,0),(\pi, 0),\left(\theta_{1}, 0\right)$ e $\left(\theta_{2}, 0\right)$ onde $\pi<\theta_{1}<\frac{3 \pi}{2}$ e $\frac{3 \pi}{2}<\theta_{2}<2 \pi$ são soluções de $s+2 c^{2}=0$.

Temos que

$$
D X(\theta, 0)=\left[\begin{array}{ll}
-4 s c-4 c^{3}+8 c s^{2} & 0 \\
0 & s c+2 c^{3}
\end{array}\right]
$$


Assim obtemos

$$
\begin{aligned}
& D X(0,0)=\left[\begin{array}{cc}
-4 & 0 \\
0 & 2 \\
4 & 0 \\
0 & -2
\end{array}\right]
\end{aligned}
$$

Isto implica que a singularidade $(0,0)$ apresent a auto-valor angular negativo e radial positivo e a singularidade $(\pi, 0)$ apresenta auto-valor angular positivo e radial negativo.

Temos que $s c+2 c^{3}=0$ em $\theta=\theta_{1}$ e em $\theta=\theta_{2}$.Assim $-4 s c-4 c^{3}+8 s^{2} c=4 c\left(c^{2}+2 s^{2}\right)$ é positivo para $\theta=\theta_{2}$ e negativo para $\theta=\theta_{1}$.

Assim temos

$$
D X\left(\theta_{1}, 0\right)=\left[\begin{array}{cc}
<0 & 0 \\
0 & 0 \\
>0 & 0 \\
0 & 0
\end{array}\right]
$$

Em $\left(\theta_{1}, 0\right)$ temos auto-valor angular negativo e radial nulo e em $\left(\theta_{2}, 0\right)$ temos auto-valor angular positivo e radial nulo.

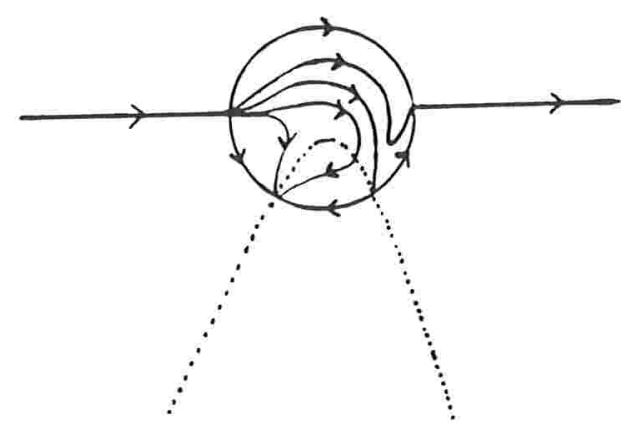

Figura 37:Desingularização em $(1,1.0,0,4)$ 


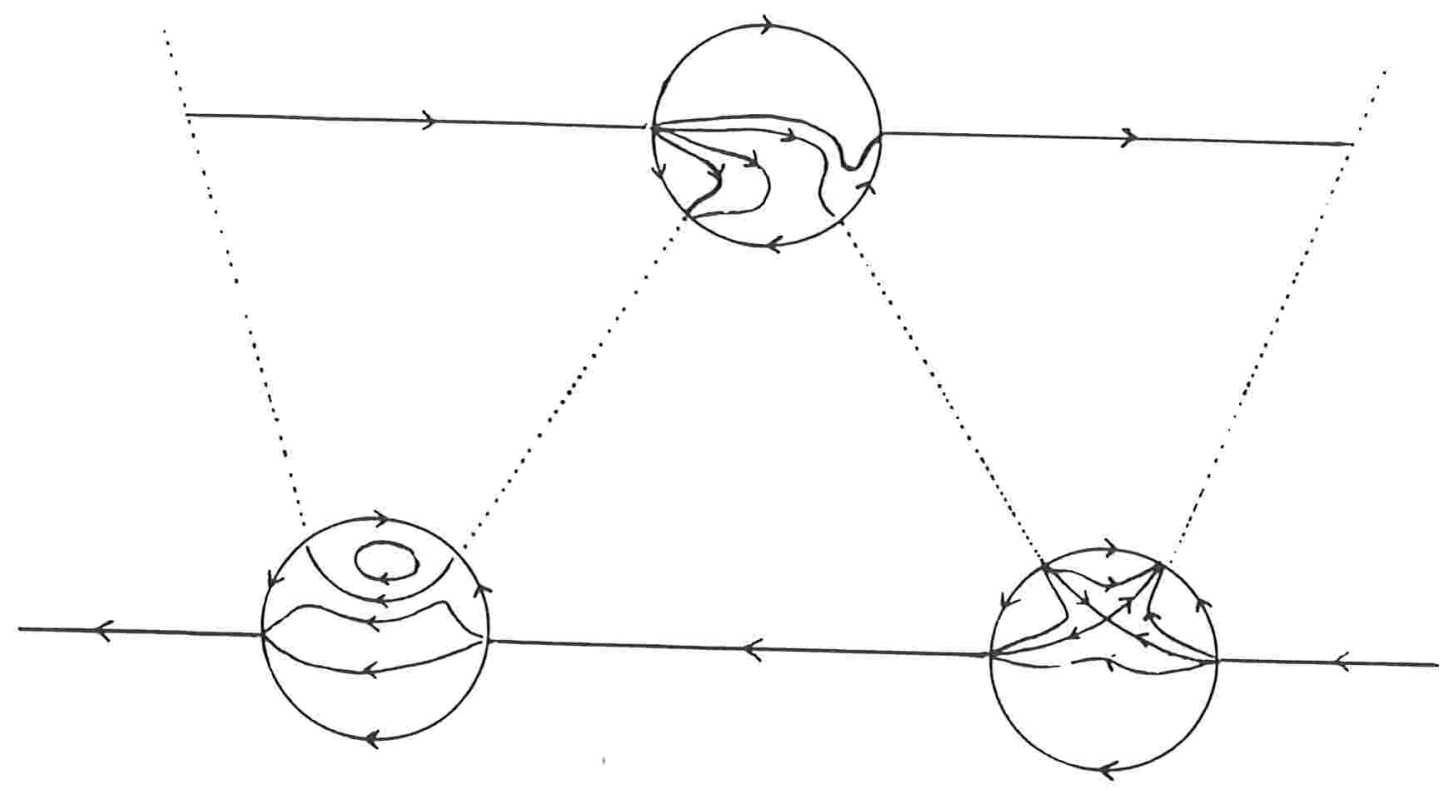

Figura 38:Desingularização Simultânea 


\section{6 - O FENÔMENO CANARD}

Tendo efetuado a desingularização de (40) estamos agora em condições de provar os teoremas 2 e 3

\section{1-Demonstraçāo do Teorema 2}

Para simplificar os cálculos transladamos o parâmetro $c(46)$ e trabalhamos com $X_{\varepsilon, b, c}$ na forma (47).

Vamos provar inicialmente que existe um Hamiltoniano $H$ com fator integrante $K$ tal que $K \bar{X}_{0}=d H, \bar{X}_{0}$ dado por (57).

Consideremos a mudança (82):

$$
\begin{aligned}
& X=\bar{x} \\
& Y=\bar{y}-12 \bar{x}^{2}
\end{aligned}
$$

Assim (57) assume a forma

$$
\left\{\begin{array}{l}
X^{\prime}=Y \\
Y^{\prime}=-2 X-24 X Y
\end{array}\right.
$$

Consideremos $F(X, Y)$ dada por

$$
F(X, Y)=144 X^{2}+(1+12 Y)-\ln (1+12 Y)
$$

Temos que $F(X, Y)$ é uma integral primeira de (83). De fato

$$
\left(F_{X}, F_{Y}\right)(Y,-2 X-24 X Y)=0
$$

Sejam $G(\bar{x}, \bar{y})$ e $H(\bar{x}, \bar{y})$ dadas por

$$
G(\bar{x}, \bar{y})=(1+12 \bar{y})-\ln \left(1+12 \bar{y}-144 \bar{x}^{2}\right)
$$




$$
H(\bar{x}, \bar{y})=[\exp (1-12 \bar{y})]\left(1+12 \bar{y}-144 \bar{x}^{2}\right)
$$

Temos

$$
\begin{aligned}
& H_{\bar{x}}=-144 e^{(1-12 \bar{y})}(2 \bar{x}) \\
& H_{\bar{y}}=-144 e^{(1-12 \bar{y})}\left(\bar{y}-12 \bar{x}^{2}\right)
\end{aligned}
$$

Assim

$$
K(\bar{x}, \bar{y})=-144 \mathrm{e}^{(1-12 \bar{y})}
$$

é o fator integrante e o Hamiltoniano $H$ é dado por (86).

Agora consideremos $P_{(1,0,0)}$ definido em 5. Temos que $P_{(1,0,0)}$ é um espaço 3-dimensional . Um ponto em $P_{(1,0,0)}$ admite 3 coordenadas $(u, v, \theta)$, indicadas na figura
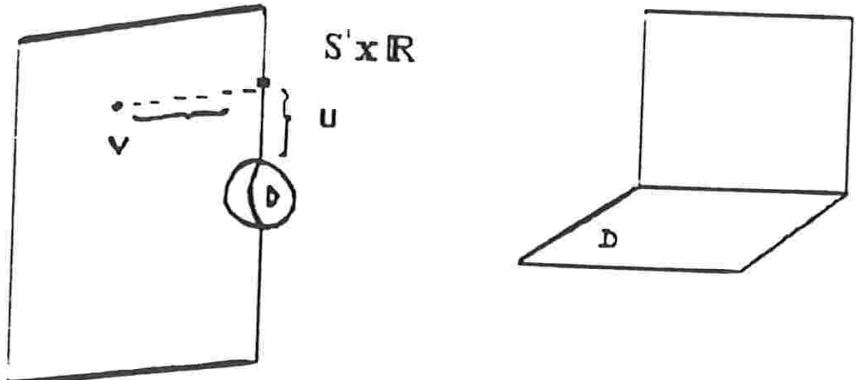

Figura 39:O Espaço $P_{(1,0,0)}$ 
Quando $u=0$, chamamos $D$ a parte correspondente a $v=0$. Seja $R$ um retângulo em $P_{(1.0 .0)}^{\prime}$ com um lado $r$ em $I$ e tal que $R$ é transversal a $\partial P$. Tomamos $R$ tal que a conexão $c$ unindo $\left(\theta_{1}, 0\right)$ a $\left(\theta_{2}, 0\right)$ é transversal a $r$ em seu ponto médio. Seja $S$ um subretängulo em $R$ com um lado $s$ em $D$ e tal que $c \cap r$ é o ponto médio de $s$.

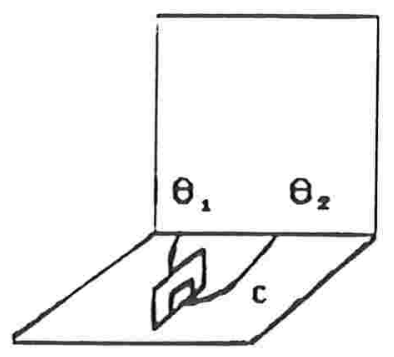

Figura 40:Os retângulos $R$ e $S$ em. $P_{(1,0,0)}$

Sejam $T$, dada por $y=32$ unindo os pontos do gráfico de $F_{0.0}$ e $N=T^{\perp}$ em $\left(0 . F_{0.0}(0\right.$,$) ,$ $T^{\perp} \subset P_{(1,0,0)}$. Seja $C_{N}$ a saturada de $N$, isto é, o fecho da união de segmentos de órbitas de $X_{(u, v, \theta)}$ por pontos de $N$ e tomados entre a primeira interseç̧ão de sua trajetória com $S$ em tempo negativo e com $R$ em tempo positivo.

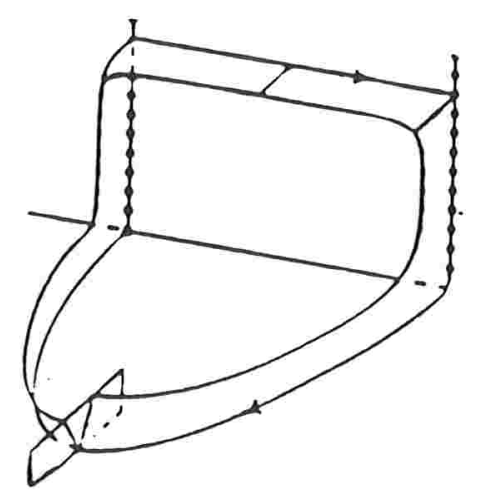

Figura 41: Variedade Central 
Seja $w_{u, \bar{b}, \bar{c}}$ a 1 -forma dual associada a (49).

$$
u_{u, \bar{b}, \bar{c}}=\left(\bar{y}-\bar{b} \bar{x}-u \overline{c x^{2}}-12 \bar{x}^{2}+4 u \bar{x}^{3}-u^{2} \bar{x}^{4}\right) d \bar{y}-\left(u \bar{x}^{2}-2 \bar{x}\right) d \bar{x}
$$

Temos

$$
-144 e^{(1-12 \bar{y})} w_{u, \bar{b}, \bar{c}}=d H(\bar{x}, \bar{y})-144 e^{(1-12 \bar{y})}\left(\left(-\bar{b} \bar{x}-u \overline{c x}^{2}+4 u \bar{x}^{3}-u^{2} \bar{x}^{4}\right) d \bar{y}-u \bar{x}^{2} d \bar{x}\right)
$$

Consideremos a seção $[\alpha, \beta]$ unindo o centro $\alpha=(0,0)$ e o ponto $\beta=\left(0,-\frac{1}{12}\right)$ na conexão $c$. Parametrizamos $[\alpha, \beta]$ pelo valor do Hamiltoniano $H$. Denotamos $C_{h}=H^{-1}(h) \operatorname{com} h \in(0, e)$ $\left(H(0,0)=e, H\left(0,-\frac{1}{12}\right)=0\right)$.

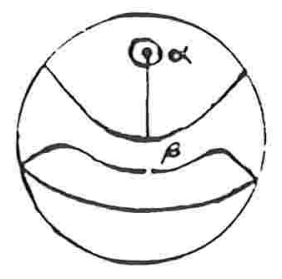

Figura 42 : Seção $[\alpha, \beta]$ em $D$

O desenvolvimento assintótico da aplicação de Poincaré $P_{u, \bar{b}, \bar{c}}$ usando o lema de Pertubação [DRS1], é dado por

$$
P_{u, \bar{b}, \bar{c}}(h)=h+\bar{b} I_{1}(h)+\bar{c} I_{2}(h)+u I_{3}(h)+o(|u, \bar{b}, \bar{c}|)
$$

onde

$$
\begin{gathered}
I_{1}(h)=144 \int_{C_{h}} \epsilon^{(1-12 \bar{y})} \bar{x} d \bar{y} \\
I_{2}(h)=144 u \int_{C_{h}} e^{(1-12 \bar{y})} \bar{x}^{2} d \bar{y}=0 \\
I_{3}(h)=-576 \int_{C_{h}} e^{(1-12 \bar{y})} \bar{x}^{3} d \bar{y}+144 \int_{C_{h}} e^{(1-12 \bar{y})} \bar{x}^{2} d \bar{x}
\end{gathered}
$$


Se $u=h=0$ o desenvolvimento de separação entre $C_{b, c}^{+}$e $C_{b, c}^{-}$(figura 47 ) é dado por

$$
\Delta(\bar{b}, \bar{c})=\bar{b} I_{1}(0)
$$

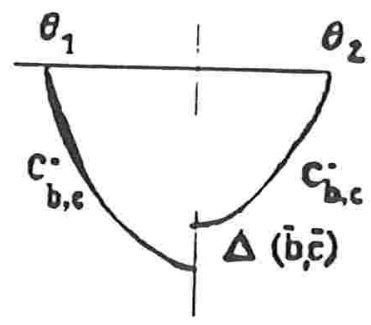

Figura 43:Separatrizes $c_{b, c}^{+}$e $c_{b, c}^{-}$

Se $h=f(u, \bar{b}, \bar{c})$ e $h=g(u, \bar{b}, \bar{c})$ denotam as intersecções de $C_{N}(\bar{b}, \bar{c})$ com $R$ então, de acordo com [DR1], temos que

$$
f(u, \bar{b}, \bar{c})-g(u, \bar{b}, \bar{c})=\bar{b} I_{1}(0)+u I_{3}(0)+o(|u, \bar{b}, \bar{c}|)
$$

Seja $\Delta(u, b, c)$ dada por

$$
\Delta(u, b, c)=f(u, \bar{b}, \bar{c})-g(u, \bar{b}, \bar{c})
$$

Para $h=0$ temos que $K(\bar{x}, \bar{y})=-144 e^{\left(2-144 x^{2}\right)}$ é uma função par e $d \bar{y}=24 \bar{x} d \bar{x}$. Utilizando integração por partes obtemos $I_{1}(0) \neq 0, I_{2}(0)=0$ e $I_{3}(0)=0$. Segue então que as equações

$$
\left\{\begin{array}{c}
\Delta=0 \\
\frac{\partial \Delta}{\partial \bar{b}} \neq 0
\end{array}\right.
$$

definem $\bar{b}=\varphi(\bar{c}, u)$ a superfície Canard. 


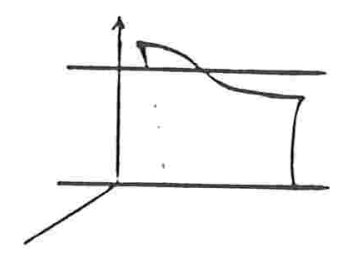

Figura 44: Superfície Canard

Para avaliarmos a estabilidade dos ciclos $\gamma$ associados aos valores dos parâmetros $(\varepsilon, b, c) \in S$ devemos calcular $\int_{\gamma} \operatorname{div} X_{\varepsilon, b, c}$.

Aproximamos $\gamma$ do LCP $\Gamma$, dado pela figura 13.

Temos

$I_{b, c}=\int_{\gamma} \operatorname{div} X_{\varepsilon, b, c} \simeq \int_{-1.3013}^{2}\left(b+2 c x-12 x^{2}+4 x^{3}\right)\left(\sqrt{1+\left(24 x-12 x^{2}+4 x^{3}\right)^{2}}+1\right) d x$

Com o auxílio do MAPLE obtemos

$$
\begin{gathered}
S_{1}=\operatorname{subs}\left(b=0.0001, c=12.0001, I_{b, c}\right) \simeq-518.12 \\
S_{2}=\operatorname{subs}\left(b=0.0001, c=11.9999, I_{b, c}\right) \simeq-523.55 \\
S_{3}=\operatorname{subs}\left(b=-0.0001, c=11.9999, I_{b, c}\right) \simeq-537.06 \\
S_{4}=\operatorname{subs}\left(b=-0.0001, c=12.0001, I_{b, c}\right) \simeq-531.63
\end{gathered}
$$

Os valores obtidos em (98) nos levam a crer que os ciclos associados aos valores dos parâmetros na superfície canard são atratores. 


\section{2-Demonstraçāo do Teorema 3}

A superfície $L$ é obtida implicitamente de maneira análoga a Superfície Canard. Para tal consideramos $\gamma=\left\{\left(2 . F_{b . c}(2)\right) \mid b . c \in R .0 \leq \varepsilon \leq \varepsilon_{0}\right\}$ formado por selas de $X_{\varepsilon . b . c}$. Saturando $\gamma$ pelo fluxo de $X_{\varepsilon, b . c}$ temos que as intersecções das variedades obtidas a partir das separatrizes definem a superfície das órbitas homoclínicas.As órbitas homoclínicas limites são dadas pela figura

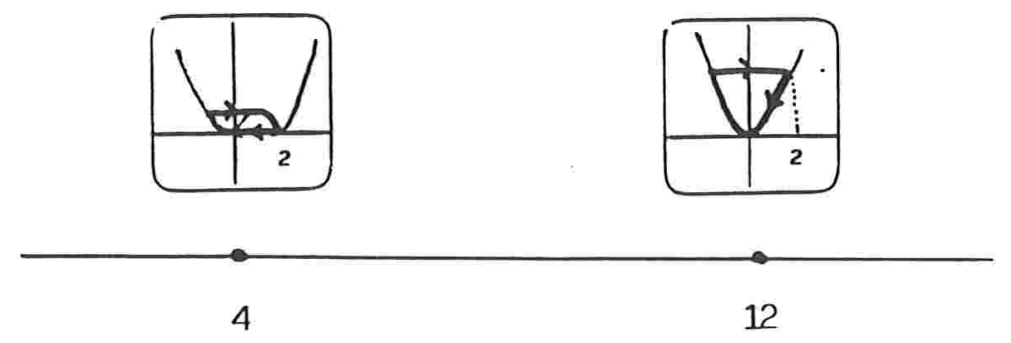

Figura 45:Órbitas Homoclínicas Limites para $\varepsilon \downarrow 0$

Seja $l(\varepsilon, b, c)$ a órbita homoclínica associada aos parâmetros $(\varepsilon, b, c)$. Temos que não existe limite de $l(\varepsilon, b, c)$, quando $\varepsilon \downarrow 0$ e $c<4$, segundo a distância de Hausdorff. Isto segue do fato que a separatriz instável não atinge a variedade lenta quando $\varepsilon \downarrow 0$.

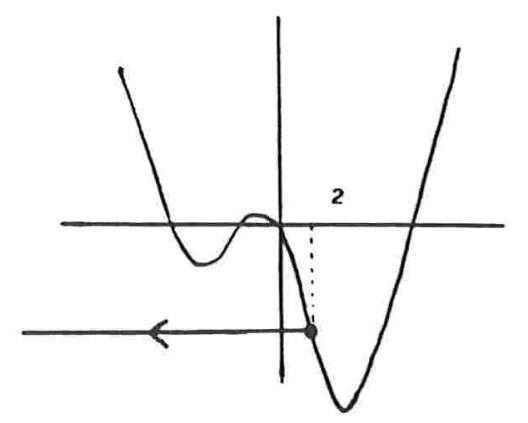

Figura 46:Não Existência de Limites Homoclínicos em $\{b=0, c<4\}$ 
Para avaliar a estabilidade da órbita homoclínica estudamos a divergência de $X_{\varepsilon, b . c}$ em $\left(2, F_{b, c}(2)\right)$. Temos que div $X_{s, b . c}\left(2, F_{b, c}(2)\right)=-F_{b, c}^{\prime}(2)=-(b+4 c-16)$. Segue então que para valores dos parâmetros em $L$ e à direita de $S C$ temos órbitas homoclínicas atratoras e à esquerda de $(S C$ ) repulsoras. Quando $\varepsilon \downarrow 0$ os valores dos parâmetros para os quais os respectivos campos admitem ciclos limites tendem para $\{b=0\}$. Assim a superfície $L$ tenderá para $\{b=0\}$. Segue que os valores dos parâmetros onde teremos órbitas homoclínicas não genéricas tenderão ao ponto $(\varepsilon, b, c)=(0,0,4)$ quando $\varepsilon \downarrow 0$. De fato, as órbitas homoclínicas não genéricas ocorrem quando os parâmetros estão em $S C$.

Precisamos avaliar o grau de degenerecência das órbitas homoclínicas correspondentes aos pontos em $£_{2}=L \cap S C$. Para isso vamos efetuar a desingularização de $X_{\varepsilon, b, c}$ em $(x, y, \varepsilon, b, c)=$ $(0,0,0,0,4)$. Efetuamos a mudança

$$
c_{1}=c-4
$$

Voltando a chamar $c$ e efetuando a mudança (48) obtemos de maneira análoga

$$
\left\{\begin{array}{l}
\bar{x}^{\prime}=\bar{y}-\bar{b} \bar{x}-4 \bar{x}^{2}-u \overline{c x}^{2}+4 u \bar{x}^{3}-u^{2} \bar{x}^{4} \\
\bar{y}^{\prime}=u \bar{x}^{2}-2 \bar{x}
\end{array}\right.
$$

Temos que $H(\bar{x}, \bar{y})$ e $K(\bar{x}, \bar{y})$ dados por (100) são tais que $K X_{0}$ é o Hamiltoniano dado por

$$
\begin{gathered}
H(\bar{x}, \bar{y})=(\exp (1-4 \bar{y}))\left(1+4 \bar{y}-16 \bar{x}^{2}\right) \\
K(\bar{x}, \bar{y})=16 \exp (1-4 \bar{y}) \\
K X_{0}:\left\{\begin{array}{l}
\bar{x}^{\prime}=-H_{\bar{y}} \\
\bar{y}^{\prime}=H_{\bar{x}}
\end{array}\right.
\end{gathered}
$$

Para avaliar o grau de degenerecência das órbitas homoclínicas utilizamos a aplicação:

$$
R(h)=\bar{b} I_{1}(h)+u \bar{c} I_{2}(h)+u I_{3}(h)+o(u, b, c)
$$


onde $I_{1}, I_{2}$ e $I_{3}$ são dados por

$$
\begin{aligned}
& I_{1}(h)=-\int_{c_{h}} K \bar{x} d \bar{y} \\
& I_{2}(h)=-\int_{c_{h}} K \bar{x}^{2} d \bar{y}=0 \\
& I_{3}(h)=\int_{c_{h}} 4 K \bar{x}^{3} d \bar{y}-\int_{c_{h}} K \bar{x}^{2} d \bar{x}
\end{aligned}
$$

A equação $R(h)=0$ define a superfície $L$. Além disso $R^{\prime}(h)$ é finito se e somente se a divergência no ponto de sela é nula $[R]$. Efetuando o cálculo obtemos $R^{\prime}(h)$ finito se e somente se $\bar{b}=-4 \bar{c}$. Para provarmos que em $\mathfrak{L}_{2}$ temos órbita homoclínica de codimensão 2 temos que provar que $R^{\prime}(h) \neq 0$. Utilizamos a aplicação $\widetilde{R}(h)=\bar{b}+u \frac{I_{3}(h)}{I_{1}(h)}$ e temos que

$$
\tilde{R}^{\prime}(h)=u\left(\frac{I_{3}(h)}{I_{1}(h)}\right)^{\prime}
$$

Com argumento similar ao utilizado em $[\mathrm{CW}]$, conforme o apêndice de [DR1] , temos que

$$
\left(\frac{I_{3}(h)}{I_{1}(h)}\right)^{\prime}<0
$$

para $h<e$. Segue que para $\bar{c}<0$ temos $\tilde{R}^{\prime}(h)<0$.

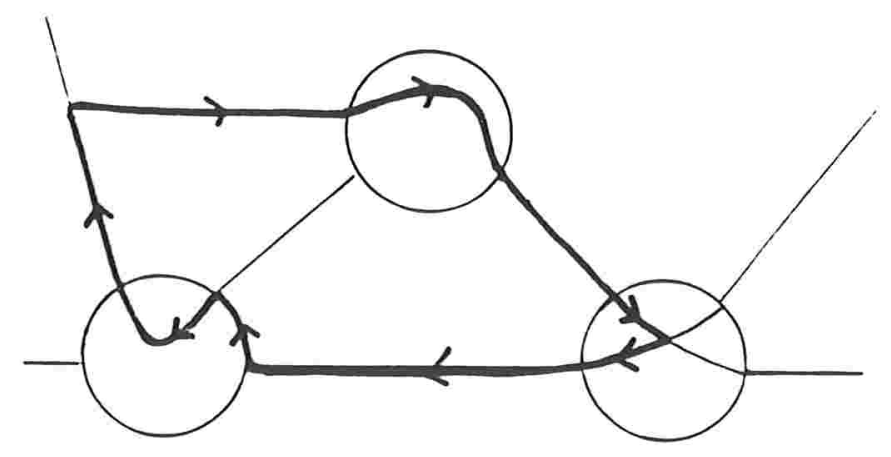

Figura 47:Órbita Homoclínica Limite de Codimensão 2

\section{3-Consideraçōes Finais}

Vamos interpretar a superfície Canard como a transição entre curvas Canard estudadas por Boutat $[\mathrm{B}]$. 
Boutat estudou o caso cúspide de codimensão 2 quando o sinal é positivo em (6) e com substituição análogas as feitas em 2 obtem-se

$$
\left\{\begin{array}{c}
x^{\prime}=y-\left(\frac{x^{2}}{2}+a x\right) \\
y^{\prime}=-\varepsilon\left(x^{2}+2 x\right)
\end{array}\right.
$$

Considerando o sinal negativo em (6) obtemos

$$
\left\{\begin{array}{l}
x^{\prime}=y-\left(-\frac{x^{2}}{2}-b x\right) \\
y^{\prime}=-\varepsilon\left(x^{2}+2 x\right)
\end{array}\right.
$$

onde

$$
a+b=2 .
$$

As curvas Canard obtidas são respectivamente
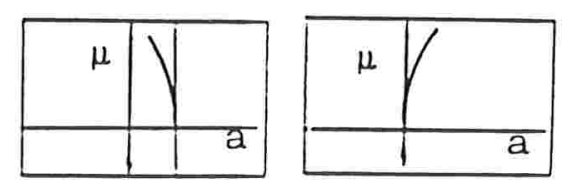

Figura 48: Curvas canard dos casos (+) e (-)

A figura (14) pode ser interpretada como a transição entre as figuras dadas em (48).

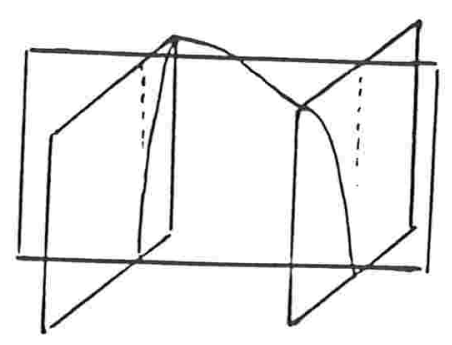

Figura 49:Transiçào entre Curvas Canard 
Outros fenômenos podem ser estudados. Com a mesma técnica se considerarmos $y=y_{0}$ com $0 \leq y_{0}<32$ obteremos uma nova superfície tal que para os parâmetros nesta superfície o LCP será dado na figura

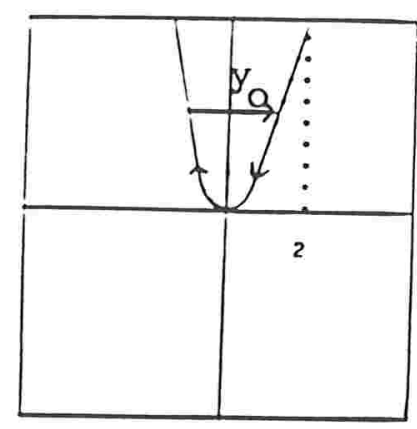

Figura 50:Ciclo Canard para $y=y_{0}<32$

A intersecção das duas superfícies Canard geram uma curva tal que para os valores do parâmetro nesta curva o par de ciclos converge para o anel dado na figura

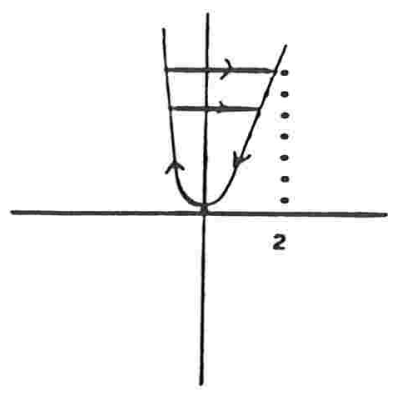

Figura 51:Anel Limite

Outras interseç̧ões podem ser estudadas e assim a distância de Hausdorff dos ciclos que formam o anel sofre significativas alterações em um vizinhança de $(0,0,12)$. 


\section{BIBLIOGRAFIA}

[AGLM] A.Andronor, E Leontovich. I.Gordon. A.Maier - Theory of bifurcations of dynamical sytems on a plane, I.P.S.T.. Jerusalem, 1971.

[B] M.A. Boutat - "Famille de Champs de Vecteurs Quadratiques du Plan de TypeBogdanov - Takens" - These - Universite de Bourgogne - 1991.

[B-1] R. Bogdanov - Versal Deformations of a Singular Point of a Vector Field on the Plane in the Case of Zero Eigenvalues. Seminar Petrovski, 1976. Sel. Math. Sov. 1 (4) - 1981.

[B-2] R. Bogdanov - Bifurcation of a Limit Cycle for a Family of Vector Fields on the Plane. - Seminar Petrovski, 1976. Sel. Math. Sov. 1 (4) - 1981.

[BCD] Benoit, E., Callot, J. L., Diener, F., Diener, M., - Chasse au Canard - Collect. Math. 31 - 32 (1 - 3) (1981).

[CW] Chow, Li and Wang -Uniqueness of Periodic Orbits in Some Vector Fields with cod.2 Singularities- JDE, 77 (2) (1989).

[D] Dumortier, F. - "Local Study of Planar Vector Fields: Singularities and their unfoldings", Stud. Math Phys. 2, Amsterdam 1991.

[DR1] Dumortier, F., Roussarie, R. - "Canard Cycles and Center Manifolds" - Memoirs of The American Mathematical Society 121(577)-1996

[DR2] Dumortier, F., Rousseau, C. - "Cubic Liénard Equations with Linear Damping," Non-Linearity 3 (1990).

[DRS-1] Dumortier, F., Roussarie, R., Sotomayor, J. - "Generic 3 - parameter families of vector fields on the plane, unfolding a singularity with nilpotent linear part - The cusp case" , Ergodic Theory Dynamical Systems 7 (1987).

[DRS-2] Dumortier, F., Roussarie, R. , Sotomayor, J. - "Generic 3 - parameter families of planar vector fields, unfoldings of saddle, focus and elliptic singularities with nilpotent linear part, in : Bifurcations of Planar Vector Fields: Nilpotent Singularities and Abelian Integrals (F. Dumortier et al., eds.), Lecture Notes in Math. 1480, Springer - Verlag, Berlin - Heidelberg - New York 1991. 
[E] W. Eckhaus, "Relaxation Oscilations Incluing a Standard Chase on French Ducks", in Asymptotic Analysis II, Springer Lect. Notes in Math. 985, 449 - 494, 1983.

[LG] R. Lutz. M. Goze - "Non Standard Analysis. A Pratical Guide with Applications". $\mathrm{LN}-881$.

$[R]$ Roussarie,R. - On the number of limit cycles which appear by perturbation of a separatix loop of planar vector fields. Bol.Soc.Brasil.Mat. 17 (1986) . 67-101.

[RW]-Roussarie,R.;Wagener,F.-A Study of The Bogdanov-Takens Bifurcation-Resenhas IMEUSP, vol.2.No.1.1995. 